\title{
Global Conservative and Multipeakon Conservative Solutions for the Modified Camassa-Holm System with Coupling Effects
}

\author{
Huabin Wen, ${ }^{1}$ Yujuan Wang, ${ }^{2}$ Yongduan Song, ${ }^{2}$ and Hamid Reza Karimi ${ }^{3}$ \\ ${ }^{1}$ School of Electrical Engineering, Beijing Jiaotong University, Beijing 100044, China \\ ${ }^{2}$ School of Automation, Chongqing University, Chongqing 400044, China \\ ${ }^{3}$ Department of Engineering, Faculty of Engineering and Science, University of Agder, 4898 Grimstad, Norway
}

Correspondence should be addressed to Yongduan Song; ydsong@cqu.edu.cn

Received 31 January 2014; Accepted 18 March 2014; Published 24 April 2014

Academic Editor: Michael Lütjen

Copyright (C) 2014 Huabin Wen et al. This is an open access article distributed under the Creative Commons Attribution License, which permits unrestricted use, distribution, and reproduction in any medium, provided the original work is properly cited.

\begin{abstract}
This paper investigates the continuation of solutions to the modified coupled two-component Camassa-Holm system after wave breaking. The underlying problem is rather challenging due to the mutual coupling effect between two components in the system. By introducing a novel transformation that makes use of a skillfully defined characteristic and a set of newly defined variables, the original system is converted into a Lagrangian equivalent system, from which the global conservative solution is obtained, which further allows for the establishment of the multipeakon conservative solution of the system. The results obtained herein are deemed useful for understanding the inevitable phenomenon near wave breaking.
\end{abstract}

\section{Introduction}

We consider here the following modified coupled twocomponent Camassa-Holm system with peakons [1]:

$$
\begin{gathered}
m_{t}+2 m u_{x}+m_{x} u+(m v)_{x}+n v_{x}=0, \quad t>0, \quad x \in R, \\
n_{t}+2 n v_{x}+n_{x} v+(n u)_{x}+m u_{x}=0, \quad t>0, x \in R, \\
m=u-u_{x x}, \quad t>0, x \in R, \\
n=v-v_{x x}, \quad t>0, \quad x \in R .
\end{gathered}
$$

System (1) is a modified version of the new coupled twocomponent Camassa-Holm system in the following equation; namely,

$$
\begin{gathered}
m_{t}=2 m u_{x}+m_{x} u+(m v)_{x}+n v_{x}, \quad t>0, x \in R, \\
n_{t}=2 n v_{x}+n_{x} v+(n u)_{x}+m u_{x}, \quad t>0, x \in R, \\
m=u-u_{x x}, \quad t>0, x \in R, \\
n=v-v_{x x}, \quad t>0, x \in R,
\end{gathered}
$$

which, as an extension of the Camassa-Holm $(\mathrm{CH})$ equation, has been established by $\mathrm{Fu}$ and $\mathrm{Qu}$ to allow for peakon solitons in the form of a superposition of multipeakons. By parameterizing $\widetilde{t}=-t$ for system (2), it then takes the form of (1), which can be rewritten as a Hamiltonian system

$$
\frac{\partial}{\partial t}\left(\begin{array}{c}
m \\
n
\end{array}\right)=-\left(\begin{array}{ll}
\partial m+m \partial & \partial m+n \partial \\
\partial n+m \partial & \partial n+n \partial
\end{array}\right)\left(\begin{array}{l}
\frac{\delta H}{\delta m}=u \\
\frac{\delta H}{\delta n}=v
\end{array}\right)
$$

with the Hamiltonian $H=(1 / 2) \int(m G * m+n G * n) d x$, where $G * m=u, G * n=v$, and $G=(1 / 2) e^{-|x|}$.Particularly, when $u=0$ (or $v=0$ ), the degenerated (1) has the same peakon solitons as the $\mathrm{CH}$ equation. We are interested in such system because it exhibits the following conserved quantities, as can be easily verified:

$$
\begin{array}{ll}
E_{1}(u)=\int_{R} u d x, & E_{2}(v)=\int_{R} v d x, \\
E_{3}(u)=\int_{R} m d x, & E_{4}(u)=\int_{R} n d x, \\
E_{5}(u, v)=\int_{R}\left(u^{2}+u_{x}^{2}+v^{2}+v_{x}^{2}\right) d x .
\end{array}
$$


Note that, when $u=v$, system (1) is reduced to the scalar Camassa-Holm equation as follows:

$$
m_{t}+4 m u_{x}+2 m_{x} u=0 \text {. }
$$

The $\mathrm{CH}$ equation, which models the unidirectional propagation of shallow water waves over a flat bottom, has a biHamiltonian structure [3] and is completely integrable [4-6]. The $\mathrm{CH}$ equation has attracted considerable attention because it has peaked solitons $[4,7]$ and experiences wave breaking $[4,8]$. The presence of breaking waves means that the solution remains bounded while its slope becomes unbounded in finite time $[8,9]$. After wave breaking, the solutions of the $\mathrm{CH}$ equation can be continued uniquely as either global conservative [10-13] or global dissipative solutions [14].

As one of the integrable multicomponent generalizations of the $\mathrm{CH}$ equation, system (1) has been shown to be locally well posed with global strong solutions which blow up in finite time $[1,2]$. Moreover, the existence issue for a class of local weak solutions for the modified coupled $\mathrm{CH} 2$ system was also addressed in [1]. It has been known that the continuation of solutions for the system beyond wave breaking has been a challenging problem. In our recent work [15], the problem of continuation beyond wave breaking for the modified coupled $\mathrm{CH} 2$ system was studied by applying an approach that reformulates the system (1) into a semilinear system of O.D.E. taking values in a Banach space. Such treatment makes it possible to investigate the continuity of the solutions beyond collision time, leading to the uniquely global solutions of this system. Also the global dissipative and multipeakon dissipative solutions of this system have been established in [16, 17], while, as far as the authors' concern, there is no effort made in the literature on the study of the global conservative as well as multipeakon conservative solutions of such system, another important feature associated with the system. Motivated by our recent work [15-17], in this paper we develop a new approach to establish a global and stable solution for the modified coupled $\mathrm{CH} 2$ system, which is conservative and further allows for the construction of the multipeakon conservative solution. The approach utilized in this paper makes use of a novel system transformation, which is different from [15] and is based on a skillfully defined characteristic and a set of newly introduced variables, where the associated energy is introduced as an additional variable so as to obtain a well-posed initial-value problem, facilitating the study on the behavior of wave breaking. It should be stressed that both global stable solution and multipeakon solution are important aspects related to the solutions near wave breaking, while there is no effort made in the literature on the study of multipeakon property of system (1), which is another motivation of this work. Our inspiration of investing the underlying issue mainly also stems from the early work $[10,11]$ in the study of the global conservative solution of the $\mathrm{CH}$ equation and [13] where the multipeakon solution is obtained for the $\mathrm{CH}$ equation. In this work a coupled system is dealt with where the mutual effect between two components makes the analysis more complicated than a single one as considered in $[10,11,13]$. By utilizing the novel transformation method, the inherent difficulty is circumvented and then the global conservative solutions of (1) are obtained, which then allows for the establishment of the multipeakon conservative solution of system (1).

The remainder of this paper is organized as follows. Section 2 presents the basic equations. In Section 3, by introducing a set of Lagrangian variables, we transform the original system into an equivalent semilinear system and derive the global solutions of the equivalent system. We obtain a global continuous semigroup of weak conservative solutions for the original system in Section 4 and the multipeakon conservative solution in Section 5.

\section{The Original System}

We first introduce an operator $\Lambda=\left(1-\partial_{x}^{2}\right)^{-1}$, which can be expressed by its associated Green's function $G=(1 / 2) e^{-|x|}$ such as $\Lambda f(x)=G * f(x)=(1 / 2) \int_{R} e^{-\left|x-x^{\prime}\right|} f\left(x^{\prime}\right) d x^{\prime}$, for all $f \in L^{2}(R)$, where $*$ denotes the spatial convolution. Thus we can rewrite (1) as a form of a quasilinear evolution equation:

$$
\begin{aligned}
& u_{t}+(u+v) u_{x}+G *\left(u v_{x}\right)+\partial_{x} G \\
& \quad *\left(u^{2}+\frac{1}{2} u_{x}^{2}+u_{x} v_{x}+\frac{1}{2} v^{2}-\frac{1}{2} v_{x}^{2}\right)=0, \quad t>0, x \in R, \\
& v_{t}+(u+v) v_{x}+G *\left(u_{x} v\right)+\partial_{x} G \\
& \quad *\left(v^{2}+\frac{1}{2} v_{x}^{2}+u_{x} v_{x}+\frac{1}{2} u^{2}-\frac{1}{2} u_{x}^{2}\right)=0, \quad t>0, x \in R .
\end{aligned}
$$

Let us define $P_{1}, P_{2}, P_{3}$, and $P_{4}$ as

$$
\begin{aligned}
P_{1}(t, x)=G *\left(u v_{x}\right)=\frac{1}{2} \int_{R} e^{-\left|x-x^{\prime}\right|}\left(u v_{x}\right)\left(t, x^{\prime}\right) d x^{\prime}, \\
P_{2}(t, x)=G *\left(u^{2}+\frac{1}{2} u_{x}^{2}+u_{x} v_{x}+\frac{1}{2} v^{2}-\frac{1}{2} v_{x}^{2}\right) \\
=\frac{1}{2} \int_{R} e^{-\left|x-x^{\prime}\right|}\left(u^{2}+\frac{1}{2} u_{x}^{2}+u_{x} v_{x}+\frac{1}{2} v^{2}-\frac{1}{2} v_{x}^{2}\right) \\
\quad \times\left(t, x^{\prime}\right) d x^{\prime}, \\
P_{3}(t, x)=G *\left(v u_{x}\right)=\frac{1}{2} \int_{R} e^{-\left|x-x^{\prime}\right|}\left(v u_{x}\right)\left(t, x^{\prime}\right) d x^{\prime}, \\
P_{4}(t, x)=G *\left(v^{2}+\frac{1}{2} v_{x}^{2}+u_{x} v_{x}+\frac{1}{2} u^{2}-\frac{1}{2} u_{x}^{2}\right) \\
=\frac{1}{2} \int_{R} e^{-\left|x-x^{\prime}\right|}\left(v^{2}+\frac{1}{2} v_{x}^{2}+u_{x} v_{x}+\frac{1}{2} u^{2}-\frac{1}{2} u_{x}^{2}\right) \\
\quad \times\left(t, x^{\prime}\right) d x^{\prime} .
\end{aligned}
$$

Then (1) can be rewritten as

$$
\begin{array}{ll}
u_{t}+(u+v) u_{x}+P_{1}+P_{2, x}=0, & t>0, x \in R, \\
v_{t}+(u+v) v_{x}+P_{3}+P_{4, x}=0, & t>0, x \in R .
\end{array}
$$


For regular solutions, we get that the total energy

$$
E(t)=\int_{R} u^{2}(t, x)+u_{x}^{2}(t, x)+v^{2}(t, x)+v_{x}^{2}(t, x) d x
$$

is constant in time. Thus (8) possesses the $H^{1}$-norm conservation law defined as

$$
\|z\|_{H^{1}}=\|u\|_{H^{1}}+\|v\|_{H^{1}}=\left(\int_{R}\left[u^{2}+u_{x}^{2}+v^{2}+v_{x}^{2}\right] d x\right)^{1 / 2}
$$

where $z(t, x)=(u, v)(t, x)$ denotes the solution of system (8). Note that $z=(u, v) \in H^{1} \times H^{1}$, and so Young's inequality ensures that $P_{1}, P_{2}, P_{3}, P_{4} \in H^{1}$.

\section{Global Solutions of the Lagrangian Equivalent System}

We reformulate system (8) as follows. For a given initial data $y(0, \xi)$, we define the corresponding characteristic $y(t, \xi)$ as the solution of

$$
y_{t}(t, \xi)=(u+v)(t, y(t, \xi))
$$

and we define the Lagrangian cumulative energy distribution $H$ as

$$
H(t, \xi)=\int_{-\infty}^{y(t, \xi)}\left(u^{2}+u_{x}^{2}+v^{2}+v_{x}^{2}\right)(t, x) d x
$$

It is not hard to check that

$$
\begin{gathered}
\left(u^{2}+u_{x}^{2}+v^{2}+v_{x}^{2}\right)_{t}+\left((u+v)\left(u^{2}+u_{x}^{2}+v^{2}+v_{x}^{2}\right)\right)_{x} \\
=\left(u^{3}-2 u P_{2}+v^{3}-2 v P_{4}\right)_{x}
\end{gathered}
$$

Then it follows from (11) and (13) that

$$
\frac{d H}{d t}=\left[\left(u^{3}-2 u P_{2}+v^{3}-2 v P_{4}\right)(t, y(t, \xi))\right]_{-\infty}^{\xi} .
$$

Throughout the following, we use the notation

$$
\begin{aligned}
U(t, \xi) & =u(t, y(t, \xi)), & V(t, \xi) & =v(t, y(t, \xi)), \\
M(t, \xi) & =u_{x}(t, y(t, \xi)), & N(t, \xi) & =v_{x}(t, y(t, \xi)) .
\end{aligned}
$$

In the following, we drop the variable $t$ for simplification. Here, we take $y$ as an increasing function for any fixed time $t$ for granted (later on we will prove this). Then after the change of variables $x=y(t, \xi)$ and $x^{\prime}=y\left(t, \xi^{\prime}\right)$, we obtain the following expressions for $P_{i}$ and $P_{i, x}(i=1,2,3,4)$; namely,

$$
\begin{aligned}
P_{1}(t, \xi)= & P_{1}(t, y(t, \xi)) \\
= & \frac{1}{2} \int_{R} e^{-\left|y(\xi)-y\left(\xi^{\prime}\right)\right|}\left[(U N) y_{\xi}\right]\left(\xi^{\prime}\right) d \xi^{\prime} \\
P_{1, x}(t, \xi)= & P_{1, x}(t, y(t, \xi)) \\
= & -\frac{1}{2} \int_{R} \operatorname{sgn}\left(\xi-\xi^{\prime}\right) e^{-\left|y(\xi)-y\left(\xi^{\prime}\right)\right|}\left[(U N) y_{\xi}\right] \\
& \times\left(\xi^{\prime}\right) d \xi^{\prime}, \\
P_{2}(t, \xi)= & P_{2}(t, y(t, \xi)) \\
= & \frac{1}{2} \int_{R} e^{-\left|y(\xi)-y\left(\xi^{\prime}\right)\right|} \\
& \times\left[\left(U^{2}+\frac{1}{2} M^{2}+M N+\frac{1}{2} V^{2}-\frac{1}{2} N^{2}\right) y_{\xi}\right] \\
& \times\left(\xi^{\prime}\right) d \xi^{\prime},
\end{aligned}
$$

$P_{2, x}(t, \xi)=P_{2, x}(t, y(t, \xi))$

$$
=-\frac{1}{2} \int_{R} \operatorname{sgn}\left(\xi-\xi^{\prime}\right) e^{-\left|y(\xi)-y\left(\xi^{\prime}\right)\right|}
$$

$$
\cdot\left[\left(U^{2}+\frac{1}{2} M^{2}+M N+\frac{1}{2} V^{2}-\frac{1}{2} N^{2}\right) y_{\xi}\right]
$$$$
\times\left(\xi^{\prime}\right) d \xi^{\prime}
$$

$$
\begin{aligned}
P_{3}(t, \xi)= & P_{3}(t, y(t, \xi)) \\
= & \frac{1}{2} \int_{R} e^{-\left|y(\xi)-y\left(\xi^{\prime}\right)\right|}\left[(V M) y_{\xi}\right]\left(\xi^{\prime}\right) d \xi^{\prime}, \\
P_{3, x}(t, \xi)= & P_{3, x}(t, y(t, \xi)) \\
= & -\frac{1}{2} \int_{R} \operatorname{sgn}\left(\xi-\xi^{\prime}\right) e^{-\left|y(\xi)-y\left(\xi^{\prime}\right)\right|}\left[(V M) y_{\xi}\right] \\
& \times\left(\xi^{\prime}\right) d \xi^{\prime}, \\
P_{4}(t, \xi)= & P_{4}(t, y(t, \xi)) \\
= & \frac{1}{2} \int_{R} e^{-\left|y(\xi)-y\left(\xi^{\prime}\right)\right|} \\
& \times\left[\left(V^{2}+\frac{1}{2} N^{2}+M N+\frac{1}{2} U^{2}-\frac{1}{2} M^{2}\right) y_{\xi}\right] \\
& \times\left(\xi^{\prime}\right) d \xi^{\prime},
\end{aligned}
$$

$$
\begin{aligned}
P_{4, x}(t, \xi)=P_{4, x}( & t, y(t, \xi)) \\
=-\frac{1}{2} \int_{R} & \operatorname{sgn}\left(\xi-\xi^{\prime}\right) e^{-\left|y(\xi)-y\left(\xi^{\prime}\right)\right|} \\
& \cdot\left[\left(V^{2}+\frac{1}{2} N^{2}+M N+\frac{1}{2} U^{2}-\frac{1}{2} M^{2}\right) y_{\xi}\right] \\
& \times\left(\xi^{\prime}\right) d \xi^{\prime} .
\end{aligned}
$$


Since $H_{\xi}=\left(u^{2}+u_{x}^{2}+v^{2}+v_{x}^{2}\right) \circ y y_{\xi}$, then $P_{2}, P_{2, x}, P_{4}$, and $P_{4, x}$ can be rewritten as

$$
\begin{aligned}
& P_{2}(t, \xi)=P_{2}(t, y(\xi)) \\
& =\frac{1}{4} \int_{R} e^{-\left|y(\xi)-y\left(\xi^{\prime}\right)\right|}\left[H_{\xi}+\left(U^{2}+2 M N-N^{2}\right) y_{\xi}\right] \\
& \times\left(\xi^{\prime}\right) d \xi^{\prime}, \\
& P_{2, x}(t, \xi)=P_{2, x}(t, y(\xi)) \\
& =-\frac{1}{4} \int_{R} \operatorname{sgn}\left(\xi-\xi^{\prime}\right) e^{-\left|y(\xi)-y\left(\xi^{\prime}\right)\right|} \\
& \times\left[H_{\xi}+\left(U^{2}+2 M N-N^{2}\right) y_{\xi}\right] \\
& \times\left(\xi^{\prime}\right) d \xi^{\prime} \\
& P_{4}(t, \xi)=P_{4}(t, y(\xi)) \\
& =\frac{1}{4} \int_{R} e^{-\left|y(\xi)-y\left(\xi^{\prime}\right)\right|}\left[H_{\xi}+\left(V^{2}+2 M N-M^{2}\right) y_{\xi}\right] \\
& \times\left(\xi^{\prime}\right) d \xi^{\prime} \\
& P_{4, x}(t, \xi)=P_{4, x}(t, y(\xi)) \\
& =-\frac{1}{4} \int_{R} \operatorname{sgn}\left(\xi-\xi^{\prime}\right) e^{-\left|y(\xi)-y\left(\xi^{\prime}\right)\right|} \\
& \times\left[H_{\xi}+\left(V^{2}+2 M N-M^{2}\right) y_{\xi}\right] \times\left(\xi^{\prime}\right) d \xi^{\prime} .
\end{aligned}
$$

From the definition of the characteristic, it is not hard to check that

$$
\begin{aligned}
U_{t}(t, \xi)= & u_{t}(t, y)+u_{x}(t, y) y_{t}(t, \xi) \\
= & \left(-P_{1}-P_{2, x}\right) \circ y(t, \xi), \\
V_{t}(t, \xi)= & v_{t}(t, y)+v_{x}(t, y) y_{t}(t, \xi) \\
= & \left(-P_{3}-P_{4, x}\right) \circ y(t, \xi), \\
M_{t}(t, \xi)= & u_{x t}(t, y)+u_{x x}(t, y) y_{t}(t, \xi) \\
= & \left(-\frac{1}{2} M^{2}-\frac{1}{2} N^{2}+U^{2}+\frac{1}{2} V^{2}-P_{1, x}-P_{2}\right) \\
& \circ y(t, \xi), \\
N_{t}(t, \xi)= & v_{x t}(t, y)+v_{x x}(t, y) y_{t}(t, \xi) \\
= & \left(-\frac{1}{2} N^{2}-\frac{1}{2} M^{2}+V^{2}+\frac{1}{2} U^{2}-P_{3, x}-P_{4}\right) \\
& \circ y(t, \xi) .
\end{aligned}
$$

We introduce another variable $\varsigma(t, \xi)$ with $\varsigma(t, \xi)=y(t, \xi)-\xi$. It will turn out that $\varsigma \in L^{\infty}(R)$. With these new variables, we now derive an equivalent system of (8) as follows:

$$
\begin{gathered}
\varsigma_{t}=U+V, \\
U_{t}=-P_{1}-P_{2, x}, \\
V_{t}=-P_{3}-P_{4, x} \\
M_{t}=\left(-\frac{1}{2} M^{2}-\frac{1}{2} N^{2}+U^{2}+\frac{1}{2} V^{2}-P_{1, x}-P_{2}\right), \\
N_{t}=\left(-\frac{1}{2} N^{2}-\frac{1}{2} M^{2}+V^{2}+\frac{1}{2} U^{2}-P_{3, x}-P_{4}\right), \\
H_{t}=U^{3}-2 U P_{2}+V^{3}-2 V P_{4},
\end{gathered}
$$

where $P_{1}$ and $P_{3}$ are given in (18), while $P_{2}, P_{2, x}, P_{4}$, and $P_{4, x}$ are given in (19). We regard system (21) as a system of ordinary differential equations in the Banach space

$$
E=W \times H^{1} \times H^{1} \times L^{2} \times L^{2} \times W
$$

endowed with the norm

$$
\|X\|_{E}=\|\varsigma\|_{W}+\|U\|_{H^{1}}+\|V\|_{H^{1}}+\|M\|_{L^{2}}+\|N\|_{L^{2}}+\|H\|_{W},
$$

for any $X=(\varsigma, U, V, M, N, H) \in E$. Here $W=\{f \in C(R) \cap$ $\left.L^{\infty}(R) \mid f_{\xi} \in L^{2}(R)\right\}$ is a Banach space with the norm given by $\|f\|_{W}=\|f\|_{L^{\infty}(R)}+\left\|f_{\xi}\right\|_{L^{2}(R)}$. Note that $H^{1}(R) \subset W$.

Differentiating (21) with respect to the variable $\xi$ yields

$$
\begin{gathered}
\varsigma_{\xi t}=U_{\xi}+V_{\xi}, \\
U_{\xi t}=\frac{1}{2} H_{\xi}+\left(\frac{1}{2} U^{2}+M N-N^{2}-P_{2}-P_{1, x}\right) y_{\xi}, \\
V_{\xi t}=\frac{1}{2} H_{\xi}+\left(\frac{1}{2} V^{2}+M N-M^{2}-P_{4}-P_{3, x}\right) y_{\xi}, \\
H_{\xi t}=\left(3 U^{2}-2 P_{2}\right) U_{\xi}-2 U P_{2, x} y_{\xi} \\
+\left(3 V^{2}-2 P_{4}\right) V_{\xi}-2 V P_{4, x} y_{\xi}
\end{gathered}
$$

which are semilinear with respect to the variables $y_{\xi}, U_{\xi}, V_{\xi}$, and $H_{\xi}$.

To obtain the uniqueness of solutions, one proceeds as follows. By proving that all functions on the right-hand side of (21) are locally Lipschitz continuous, the local existence of solutions will follow from the standard theory of ordinary differential equations in Banach spaces. In a second step, we will then prove that this local solution can be extended globally in time. Note that global solutions of (21) may not exist for all initial data in $E$. However they exist when the initial data $\bar{X}=(\bar{\varsigma}, \bar{U}, \bar{V}, \bar{M}, \bar{N}, \bar{H})$ belongs to the set $\Gamma$ which is defined as follows.

Definition 1. The set $\Gamma$ is composed of all $X=(\varsigma, U, V, M, N$, $H) \in E$ such that 
(i)

$$
(\varsigma, U, V, H) \in\left[W^{1, \infty}(R)\right]^{4},
$$

(ii)

$$
\begin{gathered}
y_{\xi} \geq 0, H_{\xi} \geq 0, \quad y_{\xi}+H_{\xi}>0 \quad \text { a.e., } \\
\lim _{\xi \rightarrow-\infty} H(\xi)=0,
\end{gathered}
$$

(iii)

$$
y_{\xi} H_{\xi}=y_{\xi}^{2} U^{2}+U_{\xi}^{2}+y_{\xi}^{2} V^{2}+V_{\xi}^{2} \quad \text { a.e., }
$$

where $W^{1, \infty}(R)=\left\{f \in C(R) \cap L^{\infty}(R) \mid f_{\xi} \in L^{\infty}(R)\right\}$ and $y(\xi)=\varsigma(\xi)+\xi$.

Lemma 2. Let $\Re_{1}: E \rightarrow W$ and let $\Re_{2}: E \rightarrow H^{1}$, or let $\Re_{2}: E \rightarrow W$ be two locally Lipschitz maps. Then, the product $X \rightarrow \mathfrak{R}_{1}(X) \Re_{2}(X)$ is also a locally Lipschitz map from $E$ to $H^{1}$ or from $E$ to $W$.

Theorem 3. Given initial data $\bar{X}=(\bar{\zeta}, \bar{U}, \bar{V}, \bar{M}, \bar{N}, \bar{H}) \in E$, there exists a time $T$ depending only on $\|\bar{X}\|_{E}$ such that the system (21) admits a unique solution in $C^{1}([0, T], E)$.

Proof. To obtain the local existence of solutions, it suffices to show that $F(X)$, given by

$$
\begin{aligned}
F(X)=( & U+V,-P_{1}-P_{2, x},-P_{3}-P_{4, x},-\frac{1}{2} M^{2}-\frac{1}{2} N^{2}+U^{2} \\
& +\frac{1}{2} V^{2}-P_{1, x}-P_{2},-\frac{1}{2} N^{2}-\frac{1}{2} M^{2}+V^{2}+\frac{1}{2} U^{2} \\
& \left.-P_{3, x}-P_{4}, U^{3}-2 U P_{2}+V^{3}-2 V P_{4}\right)
\end{aligned}
$$

with $X=(\varsigma, U, V, M, N, H)$, is a Lipchitz function on any bounded set of $E$ which is a Banach space.

Our main task is to prove the Lipschitz continuity of $P_{i}$ and $P_{i, x}(i=1,2,3,4)$ given by (18) and (19) from $E$ to $H^{1}(R)$. We first prove that $P_{2, x}$ given in (19) is locally Lipschitz continuous from $E$ to $H^{1}(R)$ and the others follow in the same way. Let us write

$$
\begin{aligned}
& P_{2, x}(\xi) \\
& =-\frac{e^{-\zeta(\xi)}}{4} \int_{R} \chi_{\left\{\xi^{\prime}<\xi\right\}} e^{-\left|\xi-\xi^{\prime}\right|} e^{\varsigma\left(\xi^{\prime}\right)} \\
& \quad \times\left[H_{\xi}+\left(U^{2}+2 M N-N^{2}\right)\left(1+\varsigma_{\xi}\right)\right]\left(\xi^{\prime}\right) d \xi^{\prime} \\
& +\frac{e^{\zeta(\xi)}}{4} \int_{R} \chi_{\left\{\xi^{\prime}>\xi\right\}} e^{-\left|\xi-\xi^{\prime}\right|} e^{-\zeta\left(\xi^{\prime}\right)} \\
& \quad \times\left[H_{\xi}+\left(U^{2}+2 M N-N^{2}\right)\left(1+\varsigma_{\xi}\right)\right]\left(\xi^{\prime}\right) d \xi^{\prime},
\end{aligned}
$$

where $\chi_{\Omega}$ denotes the indicator function of a given set $\Omega$. Let

$$
\begin{aligned}
& P_{2, x}^{1}(\xi) \\
& =-\frac{e^{-\varsigma(\xi)}}{4} \int_{R} \chi_{\left\{\xi^{\prime}<\xi\right\}} e^{-\left|\xi-\xi^{\prime}\right|} e^{\varsigma\left(\xi^{\prime}\right)} \\
& \times\left[H_{\xi}+\left(U^{2}+2 M N-N^{2}\right)\left(1+\varsigma_{\xi}\right)\right]\left(\xi^{\prime}\right) d \xi^{\prime}, \\
& P_{2, x}^{2}(\xi) \\
& \begin{aligned}
=\frac{e^{\varsigma(\xi)}}{4} \int_{R} & \chi_{\left\{\xi^{\prime}>\xi \xi\right.} e^{-\left|\xi-\xi^{\prime}\right|} e^{-\varsigma\left(\xi^{\prime}\right)} \\
& \times\left[H_{\xi}+\left(U^{2}+2 M N-N^{2}\right)\left(1+\varsigma_{\xi}\right)\right]\left(\xi^{\prime}\right) d \xi^{\prime} .
\end{aligned}
\end{aligned}
$$

We rewrite $P_{2, x}^{1}(\xi)$ as

$$
P_{2, x}^{1}(\xi)=-\frac{e^{-\varsigma(\xi)}}{2} \Lambda \circ R(X)(\xi)
$$

where $R$ is the operator from $E$ to $L^{2}(R)$ given as

$$
R(X)(\xi)=\chi_{\left\{\xi^{\prime}<\xi\right\}} e^{\varsigma}\left[H_{\xi}+\left(U^{2}+2 M N-N^{2}\right)\left(1+\varsigma_{\xi}\right)\right] .
$$

Since the operator $\Lambda$ (given in Section 2) is linear and continuous from $H^{-1}(R)$ to $H^{1}(R)$ and $L^{2}(R)$ is continuously embedded in $H^{-1}(R)$, we have $\Lambda \circ R(X) \in H^{1}$. It is not hard to check that $R$ is locally Lipschitz continuous from $E$ to $L^{2}(R)$ and therefore from $E$ to $H^{-1}(R)$. Thus $\Lambda \circ R$ is locally Lipschitz continuous from $E$ to $H^{1}(R)$. Since the mapping $X \rightarrow e^{-\varsigma}$ is locally Lipschitz continuous from $E$ to $W$, by Lemma 2, we deduce that $P_{2, x}^{1}(\xi)$ is locally Lipschitz continuous from $E$ to $H^{1}(R)$. Similarly, $P_{2, x}^{2}(\xi)$ is also locally Lipschitz continuous and therefore $P_{2, x}(\xi)$ is locally Lipschitz continuous. One proceeds in the same way and proves that $P_{1}, P_{1, x}, P_{3}$, and $P_{3, x}$ defined by (18) and $P_{2}, P_{4}$, and $P_{4, x}$ defined by (19) are locally Lipschitz continuous from $E$ to $H^{1}(R)$. We rewrite the solutions of (21) as

$$
X(t)=\bar{X}+\int_{0}^{t} F(X(\tau)) d \tau .
$$

Thus the theorem follows from the standard contraction argument of ordinary differential equations.

It remains to prove the existence of global solutions of (21). Theorem 3 gives us the existence of local solutions of (21) for initial data in $E$. In the following, we will only consider initial data that belongs to $\widetilde{E}$ given by $\widetilde{E}=E \cap\left[\left(W^{1, \infty}(R)\right)^{3} \cap\right.$ $\left.\left(L^{2}\right)^{2} \cap W^{1, \infty}(R)\right]$. To obtain that the solution of (21) belongs to $\widetilde{E}$, we have to specify the initial condition for (24). Let

$$
\begin{gathered}
\Omega=\left\{\xi \in R|| \bar{\zeta}_{\xi}(\xi)\left|\leq\left\|\bar{\zeta}_{\xi}\right\|_{L^{\infty}},\right| \bar{U}_{\xi}(\xi) \mid \leq\left\|\bar{U}_{\xi}\right\|_{L^{\infty}},\right. \\
\left.\left|\bar{V}_{\xi}(\xi)\right| \leq\left\|\bar{V}_{\xi}\right\|_{L^{\infty}},\left|\bar{H}_{\xi}(\xi)\right| \leq\left\|\bar{H}_{\xi}\right\|_{L^{\infty}}\right\} .
\end{gathered}
$$


We have meas $\left(\Omega^{c}\right)=0$. For $\xi \in \Omega^{c},\left(\varsigma_{\xi}, U_{\xi}, V_{\xi}, H_{\xi}\right)(0, \xi)$ is taken as $(0,0,0,0)$, and $\left(\varsigma_{\xi}, U_{\xi}, V_{\xi}, H_{\xi}\right)(0, \xi)$ is given as $\left(\bar{\varsigma}_{\xi}, \bar{U}_{\xi}, \bar{V}_{\xi}, \bar{H}_{\xi}\right)(\xi)$, for $\xi \in \Omega$.

The global existence of the solution for initial data in $\Gamma$ relies essentially on the fact that the set $\Gamma$ is preserved by the flow as the next lemma shows.

Lemma 4. Given initial data $\bar{X}=(\bar{\varsigma}, \bar{U}, \bar{V}, \bar{M}, \bar{N}, \bar{H}) \in \Gamma$, one considers the local solution $X(t)=(\varsigma, U, V, M, N, H)(t) \epsilon$ $C([0, T], E)$ of $(21)$ with initial data $\bar{X}$ for some $T>0$. One then gets that $X(t) \in \Gamma$ for all $t \in[0, T]$. Moreover, for a.e. $t \in[0, T], y_{\xi}(t, \xi)>0$, for a.e. $\xi \in R$, and $\lim _{\xi \rightarrow \pm \infty} H(t, \xi)$ exists and is independent of time for all $t \in[0, T]$.

Proof. We first show that $X(t) \in \Gamma$ for all $t \in[0, T]$. For any given initial data $\bar{X} \in \widetilde{E}$, we get that the local solution $(\varsigma, U, V, H)(t)$ of $(21)$ belongs to $\left[W^{1, \infty}(R)\right]^{4}$, which satisfies (25) for all $t \in[0, T]$. We now show that (27) holds for any $\xi \in \Omega$ and therefore a,e.. Consider a fixed $\xi \in \Omega$ and drop it in the notation if there is no ambiguity. On the one hand, it follows from (24) that

$$
\begin{aligned}
& \left(y_{\xi} H_{\xi}\right)_{t} \\
& =y_{\xi} H_{\xi}+y_{\xi} H_{\xi t} \\
& =\left(U_{\xi}+V_{\xi}\right) H_{\xi}+y_{\xi}\left[\left(3 U^{2}-2 P_{2}\right) U_{\xi}+\left(3 V^{2}-2 P_{4}\right) V_{\xi}\right. \\
& \left.\quad-2 U P_{2, x} y_{\xi}-2 V P_{4, x} y_{\xi}\right] \\
& =U_{\xi} H_{\xi}+V_{\xi} H_{\xi}+3 U^{2} U_{\xi} y_{\xi}-2 P_{2} U_{\xi} y_{\xi}+3 V^{2} V_{\xi} y_{\xi} \\
& \quad-2 P_{4} V_{\xi} y_{\xi}-2 U P_{2, x} y_{\xi}^{2}-2 V P_{4, x} y_{\xi}^{2},
\end{aligned}
$$

and, on the other hand,

$$
\begin{aligned}
\left(y_{\xi}^{2} U^{2}\right. & \left.+U_{\xi}^{2}+y_{\xi}^{2} V^{2}+V_{\xi}^{2}\right)_{t} \\
= & 2 y_{\xi} y_{\xi t} U^{2}+2 y_{\xi}^{2} U U_{t}+2 U_{\xi} U_{\xi t}+2 y_{\xi} y_{\xi t} V^{2} \\
& +2 y_{\xi}^{2} V V_{t}+2 V_{\xi} V_{\xi t} \\
= & U_{\xi} H_{\xi}+V_{\xi} H_{\xi}+3 U^{2} U_{\xi} y_{\xi}+3 V^{2} V_{\xi} y_{\xi}-2 P_{2} U_{\xi} y_{\xi} \\
& -2 P_{4} V_{\xi} y_{\xi}-2 U P_{2, x} y_{\xi}^{2}-2 V P_{4, x} y_{\xi}^{2} .
\end{aligned}
$$

Hence, $\left(y_{\xi} H_{\xi}\right)_{t}=\left(y_{\xi}^{2} U^{2}+U_{\xi}^{2}+y_{\xi}^{2} V^{2}+V_{\xi}^{2}\right)_{t}$. Notice that $y_{\xi} H_{\xi}(0)=\left(y_{\xi}^{2} U^{2}+U_{\xi}^{2}+y_{\xi}^{2} V^{2}+V_{\xi}^{2}\right)(0)$; then $y_{\xi} H_{\xi}(t)=$ $\left(y_{\xi}^{2} U^{2}+U_{\xi}^{2}+y_{\xi}^{2} V^{2}+V_{\xi}^{2}\right)(t)$ for all $t \in[0, T]$ and (27) has been proved. We now prove the inequalities in (26). Set $t^{*}=\sup \left\{t \in[0, T] \mid y_{\xi}\left(t^{\prime}\right) \geq 0\right.$ for all $\left.t^{\prime} \in[0, t]\right\}$. Assume that $t^{*}<T$. Since $y_{\xi}(t)$ is continuous with respect to $t$, we have $y_{\xi}\left(t^{*}\right)=0$. It follows from (27) that $U_{\xi}\left(t^{*}\right)=V_{\xi}\left(t^{*}\right)=0$. Furthermore, (24) implies that $y_{\xi t}\left(t^{*}\right)=U_{\xi}\left(t^{*}\right)+V_{\xi}\left(t^{*}\right)=$ 0 and $y_{\xi t t}\left(t^{*}\right)=\left(U_{\xi t}+V_{\xi t}\right)\left(t^{*}\right)=H_{\xi}\left(t^{*}\right)$. If $H_{\xi}\left(t^{*}\right)=$ 0 , then $\left(y_{\xi}, U_{\xi}, V_{\xi}, H_{\xi}\right)\left(t^{*}\right)=(0,0,0,0)$ which implies that $\left(y_{\xi}, U_{\xi}, V_{\xi}, H_{\xi}\right)(t)=0$ for all $t \in[0, T]$ by the uniqueness of the solution of system (24). This contradicts the fact that $y_{\xi}(0)+H_{\xi}(0)>0$ for all $\xi \in \Omega$. If $H_{\xi}\left(t^{*}\right)<0$, then $y_{\xi t t}\left(t^{*}\right)<0$. Since $y_{\xi}\left(t^{*}\right)=y_{\xi t}\left(t^{*}\right)=0$, there exists a neighborhood $\omega$ of $t^{*}$ such that $y_{\xi}(t)<0$ for all $t \in \omega /\left\{t^{*}\right\}$. This contradicts the definition of $t^{*}$. Hence, $H_{\xi}\left(t^{*}\right)>0$. We now have $y_{\xi t t}\left(t^{*}\right)>0$, which conversely implies that $y_{\xi}(t)>0$ for all $t \in \omega /\left\{t^{*}\right\}$, which contradicts the fact that $t^{*}<T$. Thus we have proved $y_{\xi}(t) \geq 0$ for all $t \in[0, T]$. We now prove that $H_{\xi} \geq 0$ for all $t \in[0, T]$. This follows from (27) when $y_{\xi}(t)>0$. If $y_{\xi}(t)=0$, then $U_{\xi}(t)=V_{\xi}(t)=0$ from (27). As we have seen, $H_{\xi}<0$ would imply that $y_{\xi}\left(t^{\prime}\right)<0$ for some $t^{\prime}$ in a punctured neighborhood of $t$, which is impossible. Hence, $H_{\xi} \geq 0$ for all $t \in[0, T]$. Now we get that $y_{\xi}(t)+H_{\xi}(t) \geq 0$ for all $t \in[0, T]$. If $y_{\xi}\left(t^{\prime}\right)+H_{\xi}\left(t^{\prime}\right)=0$ for some $t^{\prime}$, it then follows that $\left(y_{\xi}, U_{\xi}, V_{\xi}, H_{\xi}\right)\left(t^{\prime}\right)=0$ which implies that $\left(y_{\xi}, U_{\xi}, V_{\xi}, H_{\xi}\right)(t)=0$ for all $t \in[0, T]$, which contradicts the fact that $y_{\xi}(0)+H_{\xi}(0)>0$ for all $\xi \in \Omega$. Hence, $y_{\xi}(t)+H_{\xi}(t)>0$. This completes the proof that $X(t) \in \Gamma$ for all $t \in[0, T]$.

We now prove that $y_{\xi}(t, \xi)>0$ for almost all $t$. Define the set $\Theta=\left\{(t, \xi) \in[0, T] \times R \mid y_{\xi}(t, \xi)=0\right\}$. It follows from Fubini's theorem that

$$
\operatorname{meas}(\Theta)=\int_{R} \operatorname{meas}\left(\Theta_{\xi}\right) d \xi=\int_{[0, T]} \operatorname{meas}\left(\Theta_{t}\right) d t,
$$

where $\Theta_{\xi}=\left\{t \in[0, T] \mid y_{\xi}(t, \xi)=0\right\}$ and $\Theta_{t}=\{\xi \in R \mid$ $\left.y_{\xi}(t, \xi)=0\right\}$. From the above proof, we know that, for all $\xi \in \Omega, \Theta_{\xi}$ consists of isolated points that are countable. This means that meas $\left(\Theta_{\xi}\right)=0$. Since meas $\left(\Omega^{c}\right)=0$, it thus follows from (37) that meas $\left(\Theta_{t}\right)=0$ for almost every $t \in[0, T]$. This implies that $y_{\xi}(t, \xi)>0$ for almost all $t$ and therefore $y(t, \xi)$ is strictly increasing and invertible with respect to $\xi$.

For any given $t \in[0, T]$, since $H_{\xi}(t) \geq 0$ and $H(t, \xi) \epsilon$ $L^{\infty}(R)$, we know that $H(t, \pm \infty)$ exist. We have the following:

$$
H(t, \xi)=H(0, \xi)+\int_{0}^{t}\left(U^{3}-2 P_{2} U+V^{3}-2 P_{4} V\right)(\tau, \xi) d \tau
$$

Let $\xi \rightarrow \pm \infty$. Since $U, V, P_{2}, P_{4}$ are bounded in $L^{\infty}([0, T] \times$ $R)$ and $\lim _{\xi \rightarrow \pm \infty} U(t, \xi)=\lim _{\xi \rightarrow \pm \infty} V(t, \xi)=0$ as $U(t, \cdot)$, $V(t, \cdot) \in H^{1}(R)$ for all $t \in[0, T],(38)$ implies that $H(t, \pm \infty)=$ $H(0, \pm \infty)$ for all $t \in[0, T]$. Since $\bar{X} \in \Gamma$, it follows that $H(0, \pm \infty)=0$ for all $t \in[0, T]$.

Theorem 5. For any initial data $\bar{X}=(\bar{y}, \bar{U}, \bar{V}, \bar{M}, \bar{N}, \bar{H}) \in$ $\Gamma$, there exists a unique global solution $X(t)=(y, U, V, M, N$, $H)(t) \in C^{1}\left(R^{+}, E\right)$ for the system (21). Moreover, for all $t \in R^{+}$, we have $X(t) \in \Gamma$, which constructs a continuous semigroup.

Proof. To ensure that the local solution $X=(\varsigma, U, V, M, N$, $H) \in C([0, T], E)$ of system (21) can be extended to a global solution, it suffices to show that

$\sup \|\varsigma(t, \cdot), U(t, \cdot), V(t, \cdot), M(t, \cdot), N(t, \cdot), H(t, \cdot)\|_{E}<\infty$. $t \in[0, T)$ 
Since $H(t, \xi)$ is an increasing function with respect to $\xi$ for all $t$ and $\lim _{\xi \rightarrow \infty} H(t, \xi)=\lim _{\xi \rightarrow \infty} H(0, \xi)$, we have $\sup _{t \in[0, T)}\|H(t, \cdot)\|_{L^{\infty}(R)}=\|\bar{H}\|_{L^{\infty}(R)}<\infty$. We now consider a fixed $t \in[0, T)$ and drop it for simplification. Since $U_{\xi}(\xi)=$ $V_{\xi}(\xi)=0$ when $y_{\xi}(\xi)=0$ and $y_{\xi}(\xi)>0$, for a. e. $\xi$, it follows from (27) that

$$
\begin{aligned}
U^{2}(\xi) & =2 \int_{-\infty}^{\xi} U\left(\xi^{\prime}\right) U_{\xi}\left(\xi^{\prime}\right) d \xi^{\prime} \\
& =2 \int_{\left\{\xi^{\prime}<\xi \mid y_{\xi}\left(\xi^{\prime}\right)>0\right\}} U\left(\xi^{\prime}\right) U_{\xi}\left(\xi^{\prime}\right) d \xi^{\prime} \\
& \leq \int_{\left\{\xi^{\prime}<\xi \mid y_{\xi}\left(\xi^{\prime}\right)>0\right\}}\left(y_{\xi} U^{2}+\frac{U_{\xi}^{2}}{y_{\xi}}\right)\left(\xi^{\prime}\right) d \xi^{\prime} \\
& \leq \int_{R} H_{\xi}\left(\xi^{\prime}\right) d \xi^{\prime}=H(\xi),
\end{aligned}
$$

which implies that

$$
\sup _{t \in[0, T)}\left\|U^{2}(t, \cdot)\right\|_{L^{\infty}} \leq \sup _{t \in[0, T)}\|H(t, \cdot)\|_{L^{\infty}(R)}=\|\bar{H}\|_{L^{\infty}(R)}<\infty,
$$

and therefore

$$
\sup _{t \in[0, T)}\|U(t, \cdot)\|_{L^{\infty}}<\infty
$$

Similarly,

$$
\sup _{t \in[0, T)}\|V(t, \cdot)\|_{L^{\infty}}<\infty .
$$

We can obtain from the governing equation (21) that

$$
|\varsigma(t, \xi)| \leq|\varsigma(0, \xi)|+\sup _{t \in[0, T)}\left(\|U(t, \cdot)\|_{L^{\infty}}+\|V(t, \cdot)\|_{L^{\infty}}\right) T,
$$

and then $\sup _{t \in[0, T)}\|\varsigma(t, \cdot)\|_{L^{\infty}}<\infty$. We can also get from the governing equation (21) that

$$
\sup _{t \in[0, T)}\|M(t, \cdot)\|_{L^{\infty}}<\infty, \quad \sup _{t \in[0, T)}\|N(t, \cdot)\|_{L^{\infty}}<\infty .
$$

From the identity $H_{\xi}=\left(U^{2}+M^{2}+V^{2}+N^{2}\right) y_{\xi}$, we can deduce that

$$
\left|\left(U^{2}+2 M N-N^{2}\right) y_{\xi}\right| \leq\left(U^{2}+M^{2}+N^{2}+N^{2}\right) y_{\xi} \leq 2 H_{\xi},
$$

which implies that

$$
\begin{aligned}
& \left|P_{2, x}\right| \\
& \quad \leq \frac{1}{2}\left|\int_{R} e^{-\left|y(\xi)-y\left(\xi^{\prime}\right)\right|}\left[H_{\xi}+\left(U^{2}+2 M N-N^{2}\right) y_{\xi}\right]\left(\xi^{\prime}\right) d \xi^{\prime}\right| \\
& \quad \leq \frac{1}{2}\left|\int_{R} e^{-\left|y(\xi)-y\left(\xi^{\prime}\right)\right|} 3 H_{\xi}\left(\xi^{\prime}\right) d \xi^{\prime}\right| \\
& \quad \leq C \sup _{t \in[0, T)}\|H(t, \cdot)\|_{L^{\infty}(R)}<\infty .
\end{aligned}
$$

Therefore, $\left\|P_{2, x}\right\|_{L^{\infty}}<\infty$. It is not hard to know that $\left\|P_{2, x}\right\|_{L^{2}} \leq$ $C\left\|e^{-y(\xi)}\right\|_{L^{2}} \cdot \sup _{t \in[0, T)}\|H(t, \cdot)\|_{L^{\infty}(R)}<\infty$. Similarly, one can obtain that the bounds hold for $P_{1}, P_{1, x}, P_{2}, P_{3}, P_{3, x}, P_{4}$, and $P_{4, x}$. Let

$$
\begin{aligned}
Z(t)= & \|U(t, \cdot)\|_{L^{2}}+\left\|U_{\xi}(t, \cdot)\right\|_{L^{2}}+\|V(t, \cdot)\|_{L^{2}} \\
& +\left\|V_{\xi}(t, \cdot)\right\|_{L^{2}}+\left\|\varsigma_{\xi}(t, \cdot)\right\|_{L^{2}}+\left\|H_{\xi}(t, \cdot)\right\|_{L^{2}} .
\end{aligned}
$$

Using the integrated version of (21) and (24), after taking the $L^{2}$-norms on both sides, we obtain

$$
Z(t) \leq Z(0)+C \int_{0}^{t} Z(\tau) d \tau .
$$

It follows from Gronwall's inequality that $\sup _{t \in[0, T)} Z(t)<\infty$. Hence, we infer that the map $S_{t}: \Gamma \rightarrow \Gamma \times R^{+}$defined as

$$
S_{t}(\bar{X})=X(t)
$$

generates a continuous semigroup from the standard theory of ordinary differential equations.

\section{Global Conservative Solutions of the Original System}

We show that the global solution of the equivalent system (21) yields a global conservative solution of the original system (8), which constructs a continuous semigroup in this section.

To obtain the global conservative solution of the original system, we have to establish the correspondence between the Lagrangian equivalent system and the original system.

Let us first introduce the subsets $F$ and $F_{\alpha}$ of $\Gamma$ given by

$$
\begin{gathered}
F=\{X=(y, U, V, M, N, H) \in \Gamma \mid y+H \in G\}, \\
F_{\alpha}=\left\{X=(y, U, V, M, N, H) \in \Gamma \mid y+H \in G_{\alpha}\right\},
\end{gathered}
$$

where $G$ is defined as

$$
\begin{aligned}
& G=\{f \text { is invertible } \mid f-I d, \\
& \left.\quad f^{-1}-I d \text { both belong to } W^{1, \infty}(R)\right\} .
\end{aligned}
$$

And, for any $\alpha>1$, the subsets $G_{\alpha}$ of $G$ are given by

$$
G_{\alpha}=\left\{f \in G \mid\|f-I d\|_{W^{1, \infty}(R)}+\left\|f^{-1}-I d\right\|_{W^{1, \infty}(R)} \leq \alpha\right\},
$$

with a useful characterization. If $f \in G_{\alpha}(\alpha \geq 0)$, then $1 /(1+$ $\alpha) \leq f_{\xi} \leq 1+\alpha$ a.e. Conversely, if $f$ is absolutely continuous, $f-I d \in L^{\infty}(R)$ and there exists $c \geq 1$ such that $1 / c \leq f_{\xi} \leq c$ a.e., and then $f \in G_{\alpha}$ for some $\alpha$ depending only on $c$ and $\|f-I d\|_{L^{\infty}(R)}$. With this useful characterization of $G_{\alpha}$, it is not hard to prove that the space $F$ is preserved by the governing equation (21). Notice that the map $(f, X) \rightarrow X \circ f$ defines a group action of $G$ on $F$; we consider the quotient space $F / G$ of $F$ with respect to the group action. The equivalence relation on $F$ is defined as follows: for any $X, X^{\prime} \in F$, if there exists 
$f \in G$ such that $X^{\prime}=X \circ f$, we claim that $X$ and $X^{\prime}$ are equivalent.

We denote the projection $\Pi: F \rightarrow F / G$ by $\Pi(X)=$ $[X]$. For any $X=(y, U, V, M, N, H) \in F$, we introduce the mapping $\Upsilon: F \rightarrow F_{0}$ given by $\Upsilon(X)=X \circ(y+H)^{-1}$. It is not hard to prove that $\Upsilon(X)=X$ when $X \in F_{0}$ and $\Upsilon(X \circ f)=\Upsilon(X)$ for any $X \in F$ and $f \in G$. Hence, we can define the map $\tilde{\Upsilon}: F / G \rightarrow F_{0}$ as $\tilde{\Upsilon}([X])=\Upsilon(X)$, for any representative $[X] \in F / G$ of $X \in F$. For any $X \in F_{0}$, we have $\tilde{\Upsilon} \circ \Pi(X)=\Upsilon(X)=X$. Hence, $\tilde{\Upsilon} \circ \Pi_{\mid F_{0}}=I d_{\mid F_{0}}$ and any topology defined on $F_{0}$ is naturally transported into $F / G$ by this isomorphism. That is, if we equip $F_{0}$ with the metric induced by the $E$-norm; that is, $d_{F_{0}}\left(X, X^{\prime}\right)=\left\|X-X^{\prime}\right\|_{E}$, for all $X, X^{\prime} \in F_{0}$, which is complete, then the topology on $F / G$ is defined by a complete metric given by $d_{F / G}\left([X],\left[X^{\prime}\right]\right)=$ $\left\|\Upsilon(X)-\Upsilon\left(X^{\prime}\right)\right\|_{E}$ for any $[X],\left[X^{\prime}\right] \in F / G$. Let us denote by $S: F \times R^{+} \rightarrow F$ the continuous semigroup which to any initial data $\bar{X} \in F$ associates the solution $X(t)$ of (21). The system (8) is invariant with respect to relabeling. That is, for any $t>0, S_{t}(X \circ f)=S_{t}(X) \circ f$, for an $X \in F$ and $f \in G$. Thus the map $\widetilde{S}_{t}: F / G \rightarrow F / G$ given by $\widetilde{S}_{t}([X])=\left[S_{t} X\right]$ is well-defined, which generates a continuous semigroup.

To obtain a semigroup of solution for (8), we have to consider the space $D$, which characterizes the solutions in the original system:

$$
\begin{array}{r}
D=\left\{(z, \mu) \mid z \in H^{1}(R) \times H^{1}(R),\right. \\
\left.\mu_{\mathrm{ac}}=\left(u^{2}+u_{x}^{2}+v^{2}+v_{x}^{2}\right) d x\right\},
\end{array}
$$

where $z=(u, v)$ and $\mu$ is a positive finite Radon measure with $\mu_{\mathrm{ac}}$ as its absolute continuous part.

We now establish a bijection between $F / G$ and $D$ to transport the continuous semigroup obtained in the Lagrangian equivalent system (functions in $F / G$ ) into the original system (functions in $D$ ).

We first introduce the mapping $L: D \rightarrow F / G$, which transforms the original system into the Lagrangian equivalent system defined as follows.

Definition 6. For any $(z, \mu) \in D$, let

$$
\begin{gathered}
y(\xi)=\sup \{y \mid \mu(-\infty, y)+y<\xi\}, \\
U(\xi)=u \circ y(\xi), \quad V(\xi)=v \circ y(\xi), \\
M(\xi)=u_{x} \circ y(\xi), \quad N(\xi)=v_{x} \circ y(\xi), \\
H(\xi)=\xi-y(\xi),
\end{gathered}
$$

with $z=(u, v)$. We define $L(z, \mu) \in F / G$ as the equivalence class of $(y, U, V, M, N, H)$.

Remark 7. From the definition of $y, U, V, M, N, H$ in (55)(57), we can check that $X=(y, U, V, M, N, H) \in E$, which also satisfies (25). Moreover, we have $y+H=I d$ from (57), which implies that $X=(y, U, V, M, N, H) \in F_{0}$.
Furthermore, if $\mu$ is absolutely continuous, then $\mu=\left(u^{2}+\right.$ $\left.u_{x}^{2}+v^{2}+v_{x}^{2}\right) d x$ and

$$
\int_{-\infty}^{y(\xi)}\left(u^{2}+u_{x}^{2}+v^{2}+v_{x}^{2}\right) d x+y(\xi)=\xi
$$

for all $\xi \in R$.

We are led to the mapping $M$, which corresponds to the transformation from the Lagrangian equivalent system into the original system. In the other direction, we obtain the energy density $\mu$ in the original system, by pushing forward by $y$ the energy density $H_{\xi} d \xi$ in the Lagrangian equivalent system, where the push-forward $f_{\#} \nu$ of a measure $\nu$ by a measurable function $f$ is defined as

$$
f_{\#} \nu(B)=v\left(f^{-1}(B)\right),
$$

for all the Borel set $B$. Give any element $[X] \in F / G$, and let $(z, \mu)$ be defined as

$$
\begin{gathered}
z(x)=Z(\xi) \quad \text { for any } \xi \text { such that } x=y(\xi), \\
\mu=y_{\#}\left(H_{\xi} d \xi\right),
\end{gathered}
$$

where $z(x)=(u, v)(x)$ and $Z(\xi)=(U, V)(\xi)$. We get that $(z, \mu) \in D$, which does not depend on the representative $X=(y, U, V, M, N, H) \in F$ of $[X]$ that we choose. We denote by $M: F / G \rightarrow D$ the map to any $[X] \in F / G$ and $(z, \mu) \in D$ given by (60)-(61), which conversely transforms the Lagrangian equivalent system into the original system.

We claim that the transformation from the original system into the Lagrangian equivalent system is a bijection.

Theorem 8. The maps $M$ and $L$ are well-defined and $L^{-1}=M$. That is,

$$
L \circ M=I d_{F / G}, \quad M \circ L=I d_{D} .
$$

Proof. Let $[X]$ in $F / G$ be given. We consider $X=(y, U, V, M$, $N, H)=\tilde{Y}([X])$ as a representative of $[X]$ and $(z, \mu)$ given by (60)-(61) for this particular $X$. From the definition of $\widetilde{Y}$, we have $X \in F_{0}$. Let $\bar{X}=(\bar{y}, \bar{U}, \bar{V}, \bar{M}, \bar{N}, \bar{H})$ be the representative of $L(z, \mu)$ in $F_{0}$ given by Definition 6 . We have to prove that $\bar{X}=X$ and therefore $L \circ M=I d_{F / G}$. Let

$$
g(x)=\sup \{\xi \in R \mid y(\xi)<x\}
$$

Using the fact that $y$ is increasing and continuous, it follows that

$$
y(g(x))=x
$$

and $y^{-1}((-\infty, x))=(-\infty, g(x))$. From (61) and since $H(-\infty)=0$, for any $x \in R$, we get the following:

$$
\mu((-\infty, x))=\int_{y^{-1}((-\infty, x))} H_{\xi} d \xi=\int_{-\infty}^{g(x)} H_{\xi} d \xi=H(g(x)) .
$$


Since $X \in F_{0}$ and $y+H=I d$, we have

$$
\mu((-\infty, x))+x=g(x) .
$$

From the definition of $\bar{y}$, it follows that

$$
\bar{y}(\xi)=\sup \{x \in R \mid g(x)<\xi\} .
$$

For any given $\xi \in R$, using the fact that $y$ is increasing and (64), it follows that $\bar{y}(\xi) \leq y(\xi)$. If $\bar{y}(\xi)<y(\xi)$, there then exists $x$ such that $\bar{y}(\xi)<x<y(\xi)$ and (67) implies that $g(x) \geq \xi$. Conversely, since $y$ is increasing, then $x=$ $y(g(x))<y(\xi)$ implies that $g(x)<\xi$, which gives us a contradiction. Hence, we have $\bar{y}=y$. Since $y+H=I d$, it follows directly from the definitions that $\bar{H}=H, \bar{U}=U, \bar{V}=$ $V, \bar{M}=M$, and $\bar{N}=N$. We thus proved that $L \circ M=I d_{F / G}$.

Given $(z, \mu)$ in $D$, we denote by $(y, U, V, M, N, H)$ the representative of $L(z, \mu)$ in $F_{0}$ given by Definition 6 . Let $(\bar{z}, \bar{\mu})=M \circ L(z, \mu)$ and $g$ be defined as before by (63). The same computation that leads to (66) now gives

$$
\bar{\mu}((-\infty, x))+x=g(x) .
$$

Given $\xi \in R$, we consider an increasing sequence $x_{i}$ converging to $y(\xi)$ which is guaranteed by (55) such that $\mu\left(\left(-\infty, x_{i}\right)\right)+x_{i}<\xi$. Let $i$ tend to infinity. Since $F(x)=\mu((-\infty, x))$ is lower semi-continuous, we have $\mu((-\infty, y(\xi)))+y(\xi) \leq \xi$. Take $\xi=g(x)$ and then we get

$$
\mu((-\infty, x))+x \leq g(x) .
$$

By the definition of $g$, there exists an increasing sequence $\xi_{i}$ converging to $g(x)$ such that $y\left(\xi_{i}\right)<x$. It follows from the definition of $y$ in (55) that $\mu((-\infty, x))+x \geq \xi_{i}$. Passing to the limit, we obtain $\mu((-\infty, x))+x \geq g(x)$ which, together with (69), yields

$$
\mu((-\infty, x))+x=g(x)
$$

We obtain that $\bar{\mu}=\mu$ by comparing (70) and (68). It is clear from the definitions that $\bar{z}=z$. Hence, $(\bar{z}, \bar{\mu})=(z, \mu)$ and $M \circ L=I d_{D}$.

The topology defined in $F / G$ can be transported into $D$, which is guaranteed by the fact that we have established a bijection between the two equivalent systems. We define the metric $d_{D}$ on $D$ as

$$
d_{D}((z, \mu),(\bar{z}, \bar{\mu}))=d_{F / G}(L(z, \mu), L(\bar{z}, \bar{\mu})),
$$

which makes the bijection $L$ between $D$ and $F / G$ into an isometry. Since $F / G$ equipped with $d_{F / G}$ is a complete metric space, $D$ equipped with the metric $d_{D}$ is also a complete metric space. For each $t \in R$, we define the mapping $T_{t}$ : $D \rightarrow D$ as

$$
T_{t}=M \widetilde{S}_{t} L
$$

Then a continuous semigroup of conservative weak solutions for the original system is obtained as the following theorem shows.
Theorem 9. Let $(\bar{z}, \bar{\mu}) \in D$ be given. If one denotes by $t \rightarrow$ $(z(t), \mu(t))=T_{t}(\bar{z}, \bar{\mu})$ the corresponding trajectory, then $z=$ $(u, v)$ is a weak solution of the modified coupled two-component Camassa-Holm equation (8), which constructs a continuous semigroup. Moreover, $\mu$ is a weak solution of the following transport equation for the energy density:

$$
\mu_{t}+[(u+v) \mu]_{x}=\left(u^{3}-2 P_{2} u+v^{3}-2 P_{4} v\right)_{x} .
$$

Furthermore, for all $t$, it holds that

$$
\mu(t)(R)=\mu(0)(R)
$$

and, for almost all $t$,

$$
\begin{aligned}
\mu(t)(R) & =\mu_{a c}(t)(R)=\|z(t)\|_{H^{1}}^{2} \\
& =\|u(t)\|_{H^{1}}^{2}+\|v(t)\|_{H^{1}}^{2}=\mu(0)(R) .
\end{aligned}
$$

Thus the unique solution described here is a conservative weak solution of the system (8).

Proof. To prove that $z=(u, v)$ is a weak solution of the original system (8), it suffices to show that, for all $\phi \epsilon$ $C^{\infty}\left(R^{+} \times R\right)$ with compact support,

$$
\begin{array}{r}
\int_{R^{+} \times R}\left[-u \phi_{t}+(u+v) u_{x} \phi\right](t, x) d x d t \\
=-\int_{R^{+} \times R}\left[\left(P_{1}+P_{2, x}\right) \phi\right](t, x) d x d t \\
\int_{R^{+} \times R}\left[-v \phi_{t}+(u+v) v_{x} \phi\right](t, x) d x d t \\
=-\int_{R^{+} \times R}\left[\left(P_{3}+P_{4, x}\right) \phi\right](t, x) d x d t,
\end{array}
$$

where $P_{1}, P_{2, x}, P_{3}$, and $P_{4, x}$ are given by (8). Let the solution $(y, U, V, M, N, H)(t)$ of (21) be a representative of $L(z(t), \mu(t))$. On the one hand, since $y(t, \xi)$ is Lipschitz continuous and invertible with respect to $\xi$, for almost all $t$, we then can use the change of variables $x=y(t, \xi)$ and obtain

$$
\begin{aligned}
\int_{R^{+} \times R}\left[-u \phi_{t}\right. & \left.+(u+v) u_{x} \phi\right](t, x) d x d t \\
=\int_{R^{+} \times R}[ & -\left(U y_{\xi}\right)(t, \xi) \phi_{t}(t, y(t, \xi)) \\
& \left.+\left((U+V) U_{\xi}\right)(t, \xi) \phi(t, y(t, \xi))\right] d \xi d t
\end{aligned}
$$

By using the identities $y_{t}=U+V$ and $y_{\xi t}=U_{\xi}+V_{\xi}$, it then follows from (21) that

$$
\begin{gathered}
\int_{R^{+} \times R}\left[-U y_{\xi} \phi_{t}(t, y)+(U+V) U_{\xi} \phi(t, y)\right] d \xi d t \\
=\frac{1}{2} \int_{R^{+} \times R^{2}}\left\{-U N y_{\xi}+\frac{1}{2} \operatorname{sgn}\left(\xi-\xi^{\prime}\right)\right. \\
\left.\times\left[H_{\xi}+\left(U^{2}+2 M N-N^{2}\right) y_{\xi}\right]\right\}\left(\xi^{\prime}\right) \\
\cdot e^{-\left|y(\xi)-y\left(\xi^{\prime}\right)\right|} \phi(t, y(\xi)) y_{\xi}(\xi) d \xi^{\prime} d \xi d t .
\end{gathered}
$$


On the other hand, using the change of variables $x=y(t, \xi)$ and $x^{\prime}=y\left(t, \xi^{\prime}\right)$ and since $y$ is increasing with respect to $\xi$, we have the following:

$$
\begin{aligned}
& -\int_{R^{+} \times R}\left[\left(P_{1}+P_{2, x}\right) \phi\right](t, x) d x d t \\
& =\frac{1}{2} \int_{R^{+} \times R^{2}}\left[-u v_{x}+\operatorname{sgn}\left(\xi-\xi^{\prime}\right)\right. \\
& \left.\quad \times\left(u^{2}+\frac{1}{2} u_{x}^{2}+u_{x} v_{x}+\frac{1}{2} v^{2}-\frac{1}{2} v_{x}^{2}\right)\right] \\
& \quad \times\left(t, y\left(\xi^{\prime}\right)\right) \cdot e^{-\left|y(\xi)-y\left(\xi^{\prime}\right)\right|} \\
& \quad \times \phi(t, y(\xi)) y_{\xi}\left(\xi^{\prime}\right) y_{\xi}(\xi) d \xi^{\prime} d \xi d t .
\end{aligned}
$$

We obtain from (27) that

$$
\begin{gathered}
-\int_{R^{+} \times R}\left[\left(P_{1}+P_{2, x}\right) \phi\right](t, x) d x d t \\
=\frac{1}{2} \int_{R^{+} \times R^{2}}\left\{-U N y_{\xi}+\frac{1}{2} \operatorname{sgn}\left(\xi-\xi^{\prime}\right)\right. \\
\left.\times\left[H_{\xi}+\left(U^{2}+2 M N-N^{2}\right) y_{\xi}\right]\right\}\left(\xi^{\prime}\right) \\
\cdot e^{-\left|y(\xi)-y\left(\xi^{\prime}\right)\right|} \phi(t, y(\xi)) y_{\xi}(\xi) d \xi^{\prime} d \xi d t .
\end{gathered}
$$

By comparing (78) and (80), we know that

$$
\begin{gathered}
\int_{R^{+} \times R}\left[-U y_{\xi} \phi_{t}(t, y)+(U+V) U_{\xi} \phi(t, y)\right] d \xi d t \\
=-\int_{R^{+} \times R}\left[\left(P_{1}+P_{2, x}\right) \phi\right](t, x) d x d t .
\end{gathered}
$$

Hence, the first identity in (76) holds. The second identity in (76) follows in the same way. One can easily check that $\mu(t)$ is solution of (73). From the definition $\mu$ in (61), we get

$$
\mu(t)(R)=\int_{R} H_{\xi} d \xi=H(t, \infty),
$$

which is constant in time from Lemma 2. Thus, we have proved (74).

Since $y_{\xi}(t, \xi)>0$ a.e., for almost every $\xi \in R$, it then follows from (27) that

$$
\begin{aligned}
\mu(t)(B) & =\int_{y^{-1}(B)} H_{\xi} d \xi \\
& =\int_{y^{-1}(B)}\left(U^{2}+\frac{U_{\xi}^{2}}{y_{\xi}^{2}}+V^{2}+\frac{V_{\xi}^{2}}{y_{\xi}^{2}}\right) y_{\xi} d \xi,
\end{aligned}
$$

for any Borel set $B$. Since $y$ is one-to-one and $u_{x} \circ y y_{\xi}=U_{\xi}$, $v_{x} \circ y y_{\xi}=V_{\xi}$ a.e. and then (83) implies that

$$
\mu(t)(B)=\int_{B}\left(u^{2}+u_{x}^{2}+v^{2}+v_{x}^{2}\right)(t, x) d x .
$$

Hence, (75) is proved (and the solution is conservative), which completes the proof.

\section{Multipeakon Conservative Solutions of the Original System}

In this section, we will derive a new system of ordinary differential equations for the multipeakon solutions which is well posed even when collisions occur, and the variables $(y, U, V, M, N, H)$ will be used to characterize multipeakons in a way that avoids the problems related to blowing up.

Solutions of the modified coupled two-component Camassa-Holm system may experience wave breaking in the sense that the solution develops singularities in finite time, while keeping the $H^{1}$-norm finite. Continuation of the solution beyond wave breaking imposes significant challenge as can be illustrated in the case of multipeakons, which are special solutions of the modified coupled two-component Camassa-Holm system of the following form:

$$
\begin{aligned}
& u(t, x)=\sum_{i=1}^{n} p_{i}(t) e^{-\left|x-q_{i}(t)\right|}, \\
& v(t, x)=\sum_{i=1}^{n} r_{i}(t) e^{-\left|x-q_{i}(t)\right|},
\end{aligned}
$$

where $\left(p_{i}(t), r_{i}(t), q_{i}(t)\right)$ satisfy the explicit system of ordinary differential equations:

$$
\begin{gathered}
\dot{p}_{i}=\sum_{j=1, i \neq j}^{n}\left(p_{i} p_{j}+r_{i} r_{j}\right) \operatorname{sgn}\left(q_{j}-q_{i}\right) e^{-\left|q_{i}-q_{j}\right|}, \\
\dot{r}_{i}=\sum_{j=1, i \neq j}^{n}\left(p_{i} p_{j}+r_{i} r_{j}\right) \operatorname{sgn}\left(q_{j}-q_{i}\right) e^{-\left|q_{i}-q_{j}\right|}, \\
\dot{q}_{i}=-\sum_{j=1}^{n}\left(p_{j}+r_{j}\right) e^{-\left|q_{i}-q_{j}\right|} .
\end{gathered}
$$

Peakons interact in a way similar to that of solitons of the $\mathrm{CH}$ equation, and wave breaking may appear when at least two of the $q_{i}$ coincide. Clearly, if the $q_{i}$ remain distinct, the system (86) allows for a global smooth solution. It is not hard to see that $z=(u, v)$ is a global weak solution of system (8) by inserting that solution into (85). In the case where $p_{i}(0)$ and $r_{i}(0)$ have the same sign for all $i=1,2, \ldots, n,(86)$ admits a unique global solution, where the $q_{i}(t)$ remain distinct and the peakons are traveling in the same direction. However, when two peakons have opposite signs, collisions may occur, and, if so, the system (86) blows up.

Let us consider initial data $\bar{z}=(\bar{u}, \bar{v})$ given by

$$
\begin{aligned}
& \bar{u}(x)=\sum_{i=1}^{n} p_{i} e^{-\left|x-\xi_{i}\right|}, \\
& \bar{v}(x)=\sum_{i=1}^{n} r_{i} e^{-\left|x-\xi_{i}\right|} .
\end{aligned}
$$

Without loss of generality, we assume that the $p_{i}$ and $r_{i}$ are all nonzero and that the $\xi_{i}$ are all distinct. The aim is to characterize the unique and global weak solution from Theorem 9 with initial data (87) explicitly. Since the variables 
$p_{i}$ and $r_{i}$ blow up at collisions, they are not appropriate to define a multipeakon in the form of (85). We consider the following characterization of multipeakons given as continuous solutions $z=(u, v)$, which are defined on intervals $\left[y_{i}, y_{i+1}\right]$ as the solutions of the Dirichlet problem

$$
z-z_{x x}=0
$$

with boundary conditions $z\left(t, y_{i}(t)\right)=z_{i}(t)$ and $z\left(t, y_{i+1}\right.$ $(t))=z_{i+1}(t)$. The variables $y_{i}$ denote the position of the peaks, and the variables $z_{i}$ denote the values of $z$ at the peaks. In the following we will show that this property persists for conservative solutions.

Let us set $A=R \backslash\left\{\xi_{1}, \ldots, \xi_{n}\right\}$. The next lemma gives us the functions $\bar{U}, \bar{V}$, and $\bar{H}$ which belong to $C^{2}(A)$ (they even belong to $\left.C^{\infty}(A)\right)$.

Lemma 10. Let $\bar{X}=(\bar{y}, \bar{U}, \bar{V}, \bar{M}, \bar{N}, \bar{H}) \in F$ such that $(\bar{y}, \bar{U}$, $\bar{V}, \bar{H}) \in\left[C^{2}(A)\right]^{4}$ is given, and then the solution $(y, U, V, H)$ of (21) with initial data $\bar{X}$ belongs to $C^{1}\left(R^{+},\left[C^{2}(A)\right]^{4}\right)$.

Proof. To prove this Lemma, one proceeds as in Theorem 3 by using the contraction argument and replacing $E$ by

$$
\bar{E}=E \cap\left[\left(C^{2}(A)\right)^{3} \cap\left(L^{2}\right)^{2} \cap C^{2}(A)\right],
$$

endowed with the norm

$$
\begin{aligned}
\|X\|_{\bar{E}}= & \|X\|_{E}+\|y-I d\|_{W^{2, \infty}(A)}+\|U\|_{W^{2, \infty}(A)} \\
& +\|V\|_{W^{2, \infty}(A)}+\|H\|_{W^{2, \infty}(A)} .
\end{aligned}
$$

Our main task is to prove the Lipschitz continuity of $P_{i}$ and $P_{i, x}(i=1,2,3,4)$ from $\bar{E}$ to $H^{1}(R) \cap C^{2}(A)$. We first show that $P_{2, x}$ is Lipschitz continuous from $\bar{E}$ to $H^{1}(R) \cap C^{2}(A)$ and the others follow in the same way. Given a bounded set $B=\left\{X \in \bar{E} \mid\|X\|_{\bar{E}} \leq C_{B}\right\}$ where $C_{B}$ is a positive constant, from Theorem 3 , we get that

$$
\left\|P_{2, x}(X)-P_{2, x}(\bar{X})\right\|_{L^{\infty}(R)} \leq C\|X-\bar{X}\|_{E} \leq C\|X-\bar{X}\|_{\bar{E}}
$$

for a constant $C$ depending only on $C_{B}$. We can compute the derivative of $P_{2, x}$ given as

$$
P_{2, x \xi}=-\frac{1}{2} H_{\xi}+\left(P_{2}-\frac{1}{2} U^{2}-M N+N^{2}\right)\left(1+\varsigma_{\xi}\right) .
$$

From Lemma 2, $P_{2, x \xi}$ is Lipschitz continuous from $\bar{E}$ to $C(A)$ and therefore $P_{2, x}$ is Lipschitz continuous from $\bar{E}$ to $C^{1}(A)$. Similarly, we obtain the same results for $P_{1}, P_{2}, P_{3}$, and $P_{4}$ and $P_{1, x}, P_{3, x}, P_{4, x}$. We can also compute the derivative of $P_{2, x \xi}$ on $A$ as follows:

$$
\begin{aligned}
P_{2, x \xi \xi}= & -\frac{1}{2} H_{\xi \xi}+\left(P_{2, x} y_{\xi}-U U_{\xi}-M_{\xi} N-M N_{\xi}+2 N N_{\xi}\right) y_{\xi} \\
& +\left(P_{2}-\frac{1}{2} U^{2}-M N+N^{2}\right) y_{\xi \xi} .
\end{aligned}
$$

Since $P_{2, x \xi \xi}$ is locally Lipschitz maps from $\bar{E}$ to $C(A)$, we then get that $P_{2, x}$ is locally Lipschitz continuous from $\bar{E}$ to $C^{2}(A)$. The same results can be obtained for the other $P_{i}$ and $P_{i, x}(i=1,2,3,4)$ by the same way. From the standard contraction argument, the local existence of solutions of (21) can be proved in $\bar{E}$. As far as global existence is concerned, $\|X\|_{W^{1, \infty}(R)}$ does not blow up for initial data in $W^{1, \infty}(R)$. For the second derivative, for any $\xi \in A$, we get that

$$
\begin{aligned}
& y_{\xi \xi t}=U_{\xi \xi}+V_{\xi \xi}, \\
& U_{\xi \xi t}=\frac{1}{2} H_{\xi \xi}+\left(\frac{1}{2} U^{2}+M N-N^{2}-P_{2}-P_{1, x}\right) y_{\xi \xi} \\
&+\left(U U_{\xi}+M_{\xi} N+M N_{\xi}-2 N N_{\xi}\right) y_{\xi} \\
&-\left(P_{2, x}+P_{1}-U N\right) y_{\xi}^{2}, \\
& V_{\xi \xi t}= \frac{1}{2} H_{\xi \xi}+\left(\frac{1}{2} V^{2}+M N-M^{2}-P_{4}-P_{3, x}\right) y_{\xi \xi} \\
&+\left(V V_{\xi}+N_{\xi} M+N M_{\xi}-2 M N_{\xi}\right) y_{\xi} \\
&-\left(P_{4, x}+P_{3}-V M\right) y_{\xi}^{2}, \\
& H_{\xi \xi t}=-\left(2 U P_{2, x}+2 V P_{4, x}\right) y_{\xi \xi}+\left(3 U^{2}-2 P_{2}\right) U_{\xi \xi} \\
&+\left(3 V^{2}-2 P_{4}\right) V_{\xi \xi}+6 U U_{\xi}^{2}-4 P_{2, x} U_{\xi} y_{\xi} \\
&+6 V V_{\xi}^{2}-4 P_{4, x} V_{\xi} y_{\xi}-2 U P_{2} y_{\xi}^{2}+U^{3} y_{\xi}^{2} \\
&+2 U M N y_{\xi}^{2}-2 U N^{2} y_{\xi}^{2}+U H_{\xi} y_{\xi}-2 V P_{4} y_{\xi}^{2} \\
&+V^{3} y_{\xi}^{2}+2 V M N y_{\xi}^{2}-2 V M^{2} y_{\xi}^{2}+V H_{\xi} y_{\xi} .
\end{aligned}
$$

The system (94) is affine with respect to $y_{\xi \xi}, U_{\xi \xi}, V_{\xi \xi}, H_{\xi \xi}$. Thus, we get that

$$
\begin{aligned}
& \left\|X_{\xi \xi}(t, \cdot)\right\|_{L^{\infty}(A)} \\
& \quad \leq\left\|X_{\xi \xi}(0, \cdot)\right\|_{L^{\infty}(A)}+C+C \int_{0}^{t}\left\|X_{\xi \xi}(\tau, \cdot)\right\|_{L^{\infty}(A)} d \tau,
\end{aligned}
$$

where $C$ is a constant depending only on $\sup _{t \in[0, T)}\|X\|_{W^{1, \infty}(R)}$ which is bounded on any time interval $[0, T)$. It follows from Gronwall's lemma that $\|X\|_{W^{2, \infty}(A)}$ does not blow up and therefore the solution is globally defined in $\bar{E}$.

We now prove that $\bar{X}=(\bar{y}, \bar{U}, \bar{V}, \bar{M}, \bar{N}, \bar{H})$ is a representative of $z=(u, v)$ in the Lagrangian system; that is, $[\bar{X}]=L(\bar{z}, \bar{\mu})$, where $\bar{X}=(\bar{y}, \bar{U}, \bar{V}, \bar{M}, \bar{N}, \bar{H})$ is given by

$$
\bar{y}(\xi)=\xi
$$

$$
\begin{array}{rlrl}
\bar{U}(\xi) & =\bar{u}(\xi), & \bar{V}(\xi) & =\bar{v}(\xi), \\
\bar{M}(\xi)=\bar{u}_{x}(\xi), & \bar{N}(\xi)=\bar{v}_{x}(\xi), \\
\bar{H}(\xi)=\int_{-\infty}^{\xi}\left(\bar{u}^{2}+\bar{u}_{x}^{2}+\bar{v}^{2}+\bar{v}_{x}^{2}\right) d x .
\end{array}
$$


We first check that $\bar{X} \in F$. Since $\bar{z}=(\bar{u}, \bar{v})$ is a multipeakon, we get that $\bar{z}=(\bar{u}, \bar{v}) \in W^{1, \infty}(R) \cap H^{1}(R)$ from (87). Hence, $\bar{U}, \bar{V}$, and $\bar{H}$ all belong to $W^{1, \infty}(R)$ while $\bar{y}-I d$ is identically zero. Due to the exponential decay of $(\bar{u}, \bar{v})$ and $\left(\bar{u}_{x}, \bar{v}_{x}\right)$ and $\bar{H}_{\xi} \in L^{\infty}(R)$, we get that $\bar{H}_{\xi} \in L^{2}(R)$. The properties (25)(27) are straightforward to check. It is not hard to check that $M([\bar{X}])=\left(\bar{z},\left(\bar{u}^{2}+\bar{u}_{x}^{2}+\bar{v}^{2}+\bar{v}_{x}^{2}\right) d x\right)$ and, therefore, since $L \circ M=I d$, we get that $[\bar{X}]=L\left(\bar{z},\left(\bar{u}^{2}+\bar{u}_{x}^{2}+\bar{v}^{2}+\bar{v}_{x}^{2}\right) d x\right)$.

Theorem 11. Let initial data be given in (87). The solution given by Theorem 9 satisfies $z-z_{x x}=0$ between the peaks.

Proof. Let us first prove that $u-u_{x x}=0$. Assuming that $y_{\xi}(t, \xi) \neq 0$, we get that

$$
u_{x} \circ y=M, \quad u_{x x} \circ y=\frac{M_{\xi}}{y_{\xi}}=\frac{\left(U_{\xi \xi} y_{\xi}-y_{\xi \xi} U_{\xi}\right)}{y_{\xi}^{3}},
$$

and therefore

$$
\left(u-u_{x x}\right) \circ y=\frac{\left(U y_{\xi}^{3}-U_{\xi \xi} y_{\xi}+y_{\xi \xi} U_{\xi}\right)}{y_{\xi}^{3}} .
$$

We set

$$
R=U y_{\xi}^{3}-U_{\xi \xi} y_{\xi}+y_{\xi \xi} U_{\xi}
$$

For a given $\xi \in A$, differentiating (99) with respect to $t$ and after using (21), (24), and (94), we obtain

$$
\begin{aligned}
\frac{d R}{d t}= & 3 U y_{\xi}^{2} y_{\xi t}+U_{t} y_{\xi}^{3}-U_{\xi \xi t} y_{\xi}-U_{\xi \xi} y_{\xi t}+y_{\xi \xi t} U_{\xi}+y_{\xi \xi} U_{\xi t} \\
= & 2 U U_{\xi} y_{\xi}^{2}+2 U V_{\xi} y_{\xi}^{2}-\frac{H_{\xi \xi} y_{\xi}}{2} \\
& -\left(M_{\xi} N+N_{\xi} M-2 N N_{\xi}\right) y_{\xi}^{2}-U_{\xi \xi} V_{\xi} \\
& +V_{\xi \xi} U_{\xi}+\frac{H_{\xi} y_{\xi \xi}}{2} \\
= & 2 U\left(U_{\xi}+V_{\xi}\right) y_{\xi}^{2}-2 N\left(M_{\xi}-N_{\xi}\right) y_{\xi}^{2} \\
& -\frac{H_{\xi \xi} y_{\xi}}{2}+\frac{H_{\xi} y_{\xi \xi}}{2} .
\end{aligned}
$$

Differentiating (27) with respect to $\xi$, we get

$$
\begin{aligned}
y_{\xi \xi} H_{\xi}+y_{\xi} H_{\xi \xi}= & 2 y_{\xi} y_{\xi \xi} U^{2}+2 y_{\xi}^{2} U U_{\xi}+2 U_{\xi} U_{\xi \xi} \\
& +2 y_{\xi} y_{\xi \xi} V^{2}+2 y_{\xi}^{2} V V_{\xi}+2 V_{\xi} V_{\xi \xi} .
\end{aligned}
$$

After inserting the value of $y_{\xi} H_{\xi \xi}$ given by (101) into (100) and multiplying the equation by $y_{\xi}$, we obtain that

$$
\begin{aligned}
y_{\xi} & \frac{d R}{d t} \\
= & U U_{\xi} y_{\xi}^{3}-U_{\xi} U_{\xi \xi} y_{\xi}+\left(H_{\xi} y_{\xi}-y_{\xi}^{2} U^{2}-y_{\xi}^{2} V^{2}-V_{\xi}^{2}\right) y_{\xi \xi} \\
& +U V_{\xi} y_{\xi}^{3}-U_{\xi \xi} V_{\xi} y_{\xi}+U_{\xi} V_{\xi} y_{\xi \xi} .
\end{aligned}
$$

It follows from (27), and since $y_{\xi t}=\left(U_{\xi}+V_{\xi}\right)$, that

$$
y_{\xi} \cdot \frac{d R}{d t}=y_{\xi t} \cdot R
$$

We claim that, for any time $t$ such that $y_{\xi}(t) \neq 0$,

$$
\frac{d}{d t}\left(\frac{R}{y_{\xi}}\right)=\frac{R_{t} y_{\xi}-y_{\xi t} R}{y_{\xi}^{2}}=0
$$

We have to prove that $R / y_{\xi}$ is $C^{1}$ in time. Since

$$
\begin{aligned}
\frac{R}{y_{\xi}} & =U y_{\xi}^{2}-U_{\xi \xi}+\frac{y_{\xi \xi} U_{\xi}}{y_{\xi}} \\
& =U y_{\xi}^{2}-U_{\xi \xi}+\frac{y_{\xi \xi} U_{\xi}}{y_{\xi}+H_{\xi}}+\frac{y_{\xi \xi} M H_{\xi}}{y_{\xi}+H_{\xi}}=\frac{J\left(X, X_{\xi}, X_{\xi \xi}\right)}{y_{\xi}+H_{\xi}}
\end{aligned}
$$

for some polynomial $J$ and $X \in C^{1}(R, \bar{E})$, we get that $X, X_{\xi}$, and $X_{\xi \xi}$ are $C^{1}$ in time. Since $X(t)$ remains in $\Gamma$, for all $t$, from (26), we have $y_{\xi}+H_{\xi}>0$ and therefore $1 /\left(y_{\xi}+H_{\xi}\right)$ is $C^{1}$ in time, which implies that $R / y_{\xi}$ is $C^{1}$ in time. Hence, it holds that

$$
R(t, \xi)=K(\xi) y_{\xi}(t, \xi)
$$

for some constant $K(\xi)$ which is independent of time, which leads to

$$
y_{\xi}^{2}\left(u-u_{x x}\right) \circ y=K(\xi)
$$

For the multipeakons at time $t=0$, we have $y(0, \xi)=\xi$ and $\left(u-u_{x x}\right)(0, \xi)=0$ for all $\xi \in A$. Hence,

$$
\frac{R}{y_{\xi}}(t, \xi)=0
$$

for all time $t$ and all $\xi \in A$. Thus, $\left(u-u_{x x}\right)(t, \xi)=0$. Similarly, $\left(v-v_{x x}\right)(t, \xi)=0$.

For solutions with multipeakon initial data, we have the following result. If $y_{\xi}(t, \xi)$ vanishes at some point $\bar{\xi}$ in the interval $\left(\xi_{i}, \xi_{i+1}\right)$, then $y_{\xi}(t, \xi)$ vanishes everywhere in $\left(\xi_{i}, \xi_{i+1}\right)$. Moreover, for given initial multipeakon solution $\bar{z}(x)=(\bar{u}, \bar{v})(x)=\left(\sum_{i=1}^{n} p_{i} e^{-\left|x-\xi_{i}\right|}, \sum_{i=1}^{n} r_{i} e^{-\left|x-\xi_{i}\right|}\right)$, let $(y, U, V$, $M, N, H)$ be the solution of system (21) with initial data $(\bar{y}, \bar{U}, \bar{V}, \bar{M}, \bar{N}, \bar{H})$ given by (96a), (96b) and (96c), and then, between adjacent peaks, if $x_{i}=y\left(t, \xi_{i}\right) \neq x_{i+1}=y\left(t, \xi_{i+1}\right)$, the solution $z(t, x)=(u, v)(t, x)$ is twice differentiable with respect to the space variable and we have $\left(z-z_{x x}\right)=0$, for $x \in\left(x_{i}, x_{i+1}\right)$.

We now start the derivation of a system of ordinary differential equations for multipeakons. 
From (21), we get that, for each $i=1,2 \ldots, n$,

$$
\begin{gathered}
\frac{d y_{i}}{d t}=u_{i}+v_{i}, \\
\frac{d u_{i}}{d t}=-P_{1, i}-P_{2, x i}, \\
\frac{d v_{i}}{d t}=-P_{3, i}-P_{4, x i}, \\
\frac{d H_{i}}{d t}=u_{i}^{3}-2 u_{i} P_{2, i}+v_{i}^{3}-2 v_{i} P_{4, i},
\end{gathered}
$$

where $\left(y_{i}, u_{i}, v_{i}, H_{i}\right)=(y, U, V, H)\left(t, \xi_{i}\right), P_{k, i}=P_{k}\left(t, \xi_{i}\right)$, and $P_{k, x i}=P_{k, x}\left(t, \xi_{i}\right),(k=1,2,3,4)$, respectively. Since the function $y(t, \cdot)$ is invertible, for almost every $t$, we can use the change of variables $x=y(t, \xi)$ such that $P_{k, i}$ and $P_{k, x i}(k=$ $1,2,3,4)$ can be rewritten as

$$
\begin{gathered}
P_{1, i}=\frac{1}{2} \int_{R} e^{-\left|y_{i}-x\right|}\left(u v_{x}\right) d x, \\
P_{1, x i}=-\frac{1}{2} \int_{R} \operatorname{sgn}\left(y_{i}-x\right) e^{-\left|y_{i}-x\right|}\left(u v_{x}\right) d x \\
P_{2, i}=\frac{1}{2} \int_{R} e^{-\left|y_{i}-x\right|}\left(u^{2}+\frac{1}{2} u_{x}^{2}+u_{x} v_{x}+\frac{1}{2} v^{2}-\frac{1}{2} v_{x}^{2}\right) d x, \\
P_{2, x i}=-\frac{1}{2} \int_{R} \operatorname{sgn}\left(y_{i}-x\right) e^{-\left|y_{i}-x\right|} \\
\times\left(u^{2}+\frac{1}{2} u_{x}^{2}+u_{x} v_{x}+\frac{1}{2} v^{2}-\frac{1}{2} v_{x}^{2}\right) d x, \\
P_{3, i}=\frac{1}{2} \int_{R} e^{-\left|y_{i}-x\right|}\left(v u_{x}\right) d x, \\
P_{3, x i}=-\frac{1}{2} \int_{R} \operatorname{sgn}\left(y_{i}-x\right) e^{-\left|y_{i}-x\right|}\left(v u_{x}\right) d x, \\
P_{4, i}=\frac{1}{2} \int_{R} e^{-\left|y_{i}-x\right|}\left(v^{2}+\frac{1}{2} v_{x}^{2}+u_{x} v_{x}+\frac{1}{2} u^{2}-\frac{1}{2} u_{x}^{2}\right) d x, \\
P_{4, x i}=\frac{1}{2} \int_{R} \operatorname{sgn}\left(y_{i}-x\right) e^{-\left|y_{i}-x\right|} \\
\times\left(v_{x}^{2}+u_{x} v_{x}+\frac{1}{2} u^{2}-\frac{1}{2} u_{x}^{2}\right) d x .
\end{gathered}
$$

Between two adjacent peaks located at $y_{i}$ and $y_{i+1}$, we know that $z=(u, v)$ satisfies $\left(z-z_{x x}\right)=0$ and therefore $z=(u, v)$ can be written as

$$
z(x)=\left(\begin{array}{l}
u(x) \\
v(x)
\end{array}\right)=\left(\begin{array}{c}
A_{i} e^{x}+B_{i} e^{-x} \\
C_{i} e^{x}+D_{i} e^{-x}
\end{array}\right)
$$

for $x \in\left[y_{i}, y_{i+1}\right], i=1,2, \ldots, n-1$, where the constants $A_{i}$, $B_{i}, C_{i}$, and $D_{i}$ depend on $u_{i}, u_{i+1}, v_{i}, v_{i+1}, y_{i}$, and $y_{i+1}$ and read

$$
\begin{aligned}
A_{i} & =\frac{e^{-\bar{y}_{i}}}{2}\left[\frac{\bar{u}_{i}}{\cosh \left(\delta y_{i}\right)}+\frac{\delta u_{i}}{\sinh \left(\delta y_{i}\right)}\right], \\
B_{i} & =\frac{e^{\bar{y}_{i}}}{2}\left[\frac{\bar{u}_{i}}{\cosh \left(\delta y_{i}\right)}-\frac{\delta u_{i}}{\sinh \left(\delta y_{i}\right)}\right], \\
C_{i} & =\frac{e^{-\bar{y}_{i}}}{2}\left[\frac{\bar{v}_{i}}{\cosh \left(\delta y_{i}\right)}+\frac{\delta v_{i}}{\sinh \left(\delta y_{i}\right)}\right], \\
D_{i} & =\frac{e^{\bar{y}_{i}}}{2}\left[\frac{\bar{v}_{i}}{\cosh \left(\delta y_{i}\right)}-\frac{\delta v_{i}}{\sinh \left(\delta y_{i}\right)}\right],
\end{aligned}
$$

where

$$
\begin{array}{rlrl}
\bar{y}_{i} & =\frac{1}{2}\left(y_{i}+y_{i+1}\right), & \delta y_{i} & =\frac{1}{2}\left(y_{i}-y_{i+1}\right), \\
\bar{u}_{i}=\frac{1}{2}\left(u_{i}+u_{i+1}\right), & \delta u_{i}=\frac{1}{2}\left(u_{i}-u_{i+1}\right), \\
\bar{v}_{i}=\frac{1}{2}\left(v_{i}+v_{i+1}\right), & \delta v_{i}=\frac{1}{2}\left(v_{i}-v_{i+1}\right) .
\end{array}
$$

Thus, the constants $A_{i}, B_{i}, C_{i}$, and $D_{i}$ uniquely determine $z=$ $(u, v)$ on the interval $\left[y_{i}, y_{i+1}\right]$, and we compute

$$
\begin{aligned}
\delta H_{i}= & H_{i+1}-H_{i} \\
= & \int_{y_{i}}^{y_{i+1}}\left(u^{2}+u_{x}^{2}+v^{2}+v_{x}^{2}\right) d x \\
= & 2 \bar{u}_{i}^{2} \tanh \left(\delta y_{i}\right)+2 \delta u_{i}^{2} \operatorname{coth}\left(\delta y_{i}\right) \\
& +2 \bar{v}_{i}^{2} \tanh \left(\delta y_{i}\right)+2 \delta v_{i}^{2} \operatorname{coth}\left(\delta y_{i}\right) \\
= & \delta H_{1 i}+\delta H_{2 i},
\end{aligned}
$$

where $\delta H_{1 i}=2 \bar{u}_{i}^{2} \tanh \left(\delta y_{i}\right)+2 \delta u_{i}^{2} \cosh \left(\delta y_{i}\right)$ and $\delta H_{2 i}=$ $2 \bar{v}_{i}^{2} \tanh \left(\delta y_{i}\right)+2 \delta v_{i}^{2} \cosh \left(\delta y_{i}\right)$. At this point, we can get some more understanding of what is happening at a time of collision. Let $t^{*}$ be a time when the two peaks located at $y_{i}$ and $y_{i+1}$ collide, that is, such that $\lim _{t \rightarrow t^{*}} \delta y_{i}(t)=0$. Since the solution $z=(u, v)$ remains in $H^{1}$ for all time, the function $z=(u, v)$ remains continuous so that we have $\lim _{t \rightarrow t^{*}} \delta u_{i}(t)=\lim _{t \rightarrow t^{*}} \delta v_{i}(t)=0$, and, when $t$ tends to $t^{*}, A_{i}, B_{i}, C_{i}$, and $D_{i}$ may have a finite limit. However, we know that the first derivative blows up, which implies that $\lim _{t \rightarrow t^{*}} B_{i}=-\lim _{t \rightarrow t^{*}} A_{i}=\infty$ and $\lim _{t \rightarrow t^{*}} D_{i}=$ $-\lim _{t \rightarrow t^{*}} C_{i}=\infty$. Thus, $\delta u_{i}$ and $\delta v_{i}$ tend to zero, respectively, but are slower than $\delta y_{i}$. Indeed, let $t$ tend to $t^{*}$ in (114), and then, to first order in $\delta y_{i}$, we obtain that

$$
\sqrt{\delta u_{i}^{2}+\delta v_{i}^{2}}=\sqrt{\frac{\delta H_{i}}{2}} \cdot \sqrt{\delta y_{i}}+\circ\left(\delta y_{i}\right)
$$


which implies that $\delta u_{i}$ and $\delta v_{i}$ tend to zero at the same rate as $\sqrt{\delta y_{i}}$. We now turn to the computation of $P_{k, i}(k=1,2,3,4)$ given by (110). Let us write $z=(u, v)$ as

$$
\begin{aligned}
z(t, x)= & (u(t, x), v(t, x)) \\
= & \left(\sum_{j=0}^{n}\left(A_{j} e^{x}+B_{j} e^{-x}\right) \chi_{\left(y_{j}, y_{j+1}\right)}(x),\right. \\
& \left.\sum_{j=0}^{n}\left(C_{j} e^{x}+D_{j} e^{-x}\right) \chi_{\left(y_{j}, y_{j+1}\right)}(x)\right) .
\end{aligned}
$$

We have sets $y_{0}=-\infty, y_{n+1}=\infty, u_{0}=u_{n+1}=0, v_{0}=v_{n+1}=$ $0, A_{0}=u_{1} e^{-y_{1}}, B_{0}=0, A_{n}=0, B_{n}=u_{n} e^{y_{n}}$, and $C_{0}=v_{1} e^{-y_{1}}$, $D_{0}=0, C_{n}=0, D_{n}=v_{n} e^{y_{n}}$. We have

$$
\begin{gathered}
u v_{x}=\sum_{j=0}^{n}\left(A_{j} C_{j} e^{2 x}-A_{j} D_{j}+B_{j} C_{j}-B_{j} D_{j} e^{-2 x}\right) \chi_{\left(y_{j}, y_{j+1}\right)}, \\
u^{2}+\frac{1}{2} u_{x}^{2}+u_{x} v_{x}+\frac{1}{2} v^{2}-\frac{1}{2} v_{x}^{2} \\
=\sum_{j=0}^{n}\left(\left(\frac{3}{2} A_{j}^{2}+A_{j} C_{j}\right) e^{2 x}\right. \\
+\left(A_{j} B_{j}-A_{j} D_{j}-B_{j} C_{j}+2 C_{j} D_{j}\right) \\
\left.+\left(\frac{3}{2} B_{j}^{2}+B_{j} D_{j}\right) e^{-2 x}\right) \chi_{\left(y_{j}, y_{j+1}\right)}, \\
v u_{x}=\sum_{j=0}^{n}\left(A_{j} C_{j} e^{2 x}-C_{j} B_{j}+D_{j} A_{j}-B_{j} D_{j} e^{-2 x}\right) \chi_{\left(y_{j}, y_{j+1}\right)}, \\
v^{2}+\frac{1}{2} v_{x}^{2}+u_{x} v_{x}+\frac{1}{2} u^{2}-\frac{1}{2} u_{x}^{2} \\
=\sum_{j=0}^{n}\left(\left(\frac{3}{2} C_{j}^{2}+A_{j} C_{j}\right) e^{2 x}\right. \\
+\left(C_{j} D_{j}-C_{j} B_{j}-D_{j} A_{j}+2 A_{j} B_{j}\right) \\
\left.+\left(\frac{3}{2} D_{j}^{2}+D_{j} B_{j}\right) e^{-2 x}\right) \chi_{\left(y_{j}, y_{j+1}\right)}
\end{gathered}
$$

We set

$$
k_{i j}= \begin{cases}-1 & \text { if } i \leq j, \\ 1 & \text { otherwise. }\end{cases}
$$

By inserting (117) into (110), we get

$$
\begin{gathered}
P_{1, i}=\frac{1}{2} \sum_{j=0}^{n} \int_{y_{j}}^{y_{j+1}} e^{-k_{i j}\left(y_{i}-x\right)} \\
\times\left(A_{j} C_{j} e^{2 x}-A_{j} D_{j}+B_{j} C_{j}-B_{j} D_{j} e^{-2 x}\right) d x, \\
P_{2, i}=\frac{1}{2} \sum_{j=0}^{n} \int_{y_{j}}^{y_{j+1}} e^{-k_{i j}\left(y_{i}-x\right)}\left(\left(\frac{3}{2} A_{j}^{2}+A_{j} C_{j}\right) e^{2 x}\right. \\
+\left(A_{j} B_{j}-A_{j} D_{j}\right. \\
\left.-B_{j} C_{j}+2 C_{j} D_{j}\right) \\
\left.+\left(\frac{3}{2} B_{j}^{2}+B_{j} D_{j}\right) e^{-2 x}\right) d x, \\
P_{3, i}=\frac{1}{2} \sum_{j=0}^{n} \int_{y_{j}}^{y_{j+1}} e^{-k_{i j}\left(y_{i}-x\right)} \\
\times\left(A_{j} C_{j} e^{2 x}-C_{j} B_{j}+D_{j} A_{j}-B_{j} D_{j} e^{-2 x}\right) d x, \\
P_{4, i}=\frac{1}{2} \sum_{j=0}^{n} \int_{y_{j}}^{y_{j+1}} e^{-k_{i j}\left(y_{i}-x\right)} \\
\times\left(\left(\frac{3}{2} C_{j}^{2}+A_{j} C_{j}\right) e^{2 x}\right. \\
+\left(C_{j} D_{j}-C_{j} B_{j}-D_{j} A_{j}+2 A_{j} B_{j}\right) \\
\left.+\left(\frac{3}{2} D_{j}^{2}+D_{j} B_{j}\right) e^{-2 x}\right) .
\end{gathered}
$$

It follows from (112) and (114) that

$$
\begin{aligned}
A_{j}^{2}=\left[\frac{e^{-\bar{y}_{j}}}{2}\left(\frac{\bar{u}_{j}}{\cosh \left(\delta y_{j}\right)}+\frac{\delta u_{j}}{\sinh \left(\delta y_{j}\right)}\right)\right]^{2} \\
=\frac{e^{-2 \bar{y}_{j}}}{\sinh ^{2}\left(2 \delta y_{j}\right)}\left[\bar{u}_{j}^{2} \sinh ^{2}\left(\delta y_{j}\right)\right. \\
+2 \bar{u}_{j} \delta u_{j} \sinh \left(\delta y_{j}\right) \cosh \left(\delta y_{j}\right) \\
\left.+\delta u_{j}^{2} \cosh ^{2}\left(\delta y_{j}\right)\right] \\
=\frac{e^{-2 \bar{y}_{j}}}{4 \sinh \left(2 \delta y_{j}\right)}\left[\delta H_{1 j}+4 \bar{u}_{j} \delta u_{j}\right],
\end{aligned}
$$

$$
\begin{aligned}
A_{j} B_{j}= & \frac{e^{-\bar{y}_{j}}}{2}\left(\frac{\bar{u}_{j}}{\cosh \left(\delta y_{j}\right)}+\frac{\delta u_{j}}{\sinh \left(\delta y_{j}\right)}\right) \\
& \cdot \frac{e^{\bar{y}_{j}}}{2}\left(\frac{\bar{u}_{j}}{\cosh \left(\delta y_{j}\right)}-\frac{\delta u_{j}}{\sinh \left(\delta y_{j}\right)}\right)
\end{aligned}
$$




$$
\begin{aligned}
= & \frac{1}{4 \sinh \left(2 \delta y_{j}\right)}\left[2 \bar{u}_{j}^{2} \tanh \left(\delta y_{j}\right)-2 \delta u_{j}^{2} \operatorname{coth}\left(\delta y_{j}\right)\right] \\
= & \frac{1}{4 \sinh \left(2 \delta y_{j}\right)}\left[4 \bar{u}_{j}^{2} \tanh \left(\delta y_{j}\right)-\delta H_{1 j}\right] \\
A_{j} C_{j}= & \frac{e^{-\bar{y}_{j}}}{2}\left(\frac{\bar{u}_{j}}{\cosh \left(\delta y_{j}\right)}+\frac{\delta u_{j}}{\sinh \left(\delta y_{j}\right)}\right) \\
& \cdot \frac{e^{-\bar{y}_{j}}}{2}\left(\frac{\bar{v}_{j}}{\cosh \left(\delta y_{j}\right)}+\frac{\delta v_{j}}{\sinh \left(\delta y_{j}\right)}\right) \\
= & \frac{e^{-2 \bar{y}_{j}}}{2 \sinh \left(2 \delta y_{j}\right)}\left[\bar{u}_{j} \bar{v}_{j} \tanh \left(\delta y_{j}\right)+\delta u_{j} \bar{v}_{j}\right. \\
& \left.+\delta v_{j} \bar{u}_{j}+\delta u_{j} \delta v_{j} \operatorname{coth}\left(\delta y_{j}\right)\right]
\end{aligned}
$$$$
A_{j} D_{j}=\frac{e^{-\bar{y}_{j}}}{2}\left(\frac{\bar{u}_{j}}{\cosh \left(\delta y_{j}\right)}+\frac{\delta u_{j}}{\sinh \left(\delta y_{j}\right)}\right)
$$$$
\cdot \frac{e^{\bar{y}_{j}}}{2}\left(\frac{\bar{v}_{j}}{\cosh \left(\delta y_{j}\right)}-\frac{\delta v_{j}}{\sinh \left(\delta y_{j}\right)}\right)
$$$$
=\frac{1}{2 \sinh \left(2 \delta y_{j}\right)}\left[\bar{u}_{j} \bar{v}_{j} \tanh \left(\delta y_{j}\right)+\delta u_{j} \bar{v}_{j}\right.
$$$$
\left.-\delta v_{j} \bar{u}_{j}-\delta u_{j} \delta v_{j} \operatorname{coth}\left(\delta y_{j}\right)\right],
$$

$$
\begin{aligned}
& B_{j} C_{j}= \frac{e^{\bar{y}_{j}}}{2}\left(\frac{\bar{u}_{j}}{\cosh \left(\delta y_{j}\right)}-\frac{\delta u_{j}}{\sinh \left(\delta y_{j}\right)}\right) \\
& \cdot \frac{e^{-\bar{y}_{j}}}{2}\left(\frac{\bar{v}_{j}}{\cosh \left(\delta y_{j}\right)}+\frac{\delta v_{j}}{\sinh \left(\delta y_{j}\right)}\right) \\
&= \frac{1}{2 \sinh \left(2 \delta y_{j}\right)}\left[\bar{u}_{j} \bar{v}_{j} \tanh \left(\delta y_{j}\right)+\delta v_{j} \bar{u}_{j}\right. \\
&\left.-\delta u_{j} \bar{v}_{j}-\delta u_{j} \delta v_{j} \operatorname{coth}\left(\delta y_{j}\right)\right],
\end{aligned}
$$

$$
\begin{aligned}
& B_{j} D_{j}= \frac{e^{\bar{y}_{j}}}{2}\left(\frac{\bar{u}_{j}}{\cosh \left(\delta y_{j}\right)}-\frac{\delta u_{j}}{\sinh \left(\delta y_{j}\right)}\right) \\
& \cdot \frac{e^{\bar{y}_{j}}}{2}\left(\frac{\bar{v}_{j}}{\cosh \left(\delta y_{j}\right)}-\frac{\delta v_{j}}{\sinh \left(\delta y_{j}\right)}\right) \\
&= \frac{e^{2 \bar{y}_{j}}}{2 \sinh \left(2 \delta y_{j}\right)}\left[\bar{u}_{j} \bar{v}_{j} \tanh \left(\delta y_{j}\right)-\delta u_{j} \bar{v}_{j}\right. \\
&\left.-\delta v_{j} \bar{u}_{j}+\delta u_{j} \delta v_{j} \operatorname{coth}\left(\delta y_{j}\right)\right],
\end{aligned}
$$

$$
\begin{aligned}
B_{j}^{2} & =\left[\frac{e^{\bar{y}_{j}}}{2}\left(\frac{\bar{u}_{j}}{\cosh \left(\delta y_{j}\right)}-\frac{\delta u_{j}}{\sinh \left(\delta y_{j}\right)}\right)\right]^{2} \\
& =\frac{e^{2 \bar{y}_{j}}}{4 \sinh \left(2 \delta y_{j}\right)}\left[\delta H_{1 j}-4 \bar{u}_{j} \delta u_{j}\right] .
\end{aligned}
$$

Thus, from (120)-(126), we can obtain that

$$
\begin{aligned}
\int_{y_{j}}^{y_{j+1}} e^{-k_{i j}\left(y_{i}-x\right)} A_{j}^{2} e^{2 x} d x & \\
=\frac{e^{-k_{i j} y_{i}} \cdot e^{k_{i j} \bar{y}_{j}}}{2\left(2+k_{i j}\right) \sinh \left(2 \delta y_{j}\right)} \sinh ( & \left.\left(2+k_{i j}\right) \delta y_{j}\right) \\
& \times\left[\delta H_{1 j}+4 \bar{u}_{j} \delta u_{j}\right],
\end{aligned}
$$

$$
\begin{aligned}
& \int_{y_{j}}^{y_{j+1}} e^{-k_{i j}\left(y_{i}-x\right)} A_{j} C_{j} e^{2 x} d x \\
& =\frac{e^{-k_{i j} y_{i}} \cdot e^{k_{i j} \bar{y}_{j}}}{\left(2+k_{i j}\right) \sinh \left(2 \delta y_{j}\right)} \sinh \left(\left(2+k_{i j}\right) \delta y_{j}\right) \\
& \quad \cdot\left[\bar{u}_{j} \bar{v}_{j} \tanh \left(\delta y_{j}\right)+\delta u_{j} \bar{v}_{j}+\delta v_{j} \bar{u}_{j}\right. \\
& \left.\quad+\delta u_{j} \delta v_{j} \operatorname{coth}\left(\delta y_{j}\right)\right] \\
& \int_{y_{j}}^{y_{j+1}} e^{-k_{i j}\left(y_{i}-x\right)} A_{j} B_{j} d x \\
& =\frac{e^{-k_{i j} y_{i}} \cdot e^{k_{i j} \bar{y}_{j}}}{2 \sinh \left(2 \delta y_{j}\right)} \sinh \left(\delta y_{j}\right) \\
& \quad \times\left[4 \bar{u}_{j}^{2} \tanh \left(\delta y_{j}\right)-\delta H_{1 j}\right]
\end{aligned}
$$

$$
\begin{aligned}
\int_{y_{j}}^{y_{j+1}} e^{-k_{i j}\left(y_{i}-x\right)} A_{j} D_{j} d x & \\
= & \frac{e^{-k_{i j} y_{i}} \cdot e^{k_{i j} \bar{y}_{j}}}{\sinh \left(2 \delta y_{j}\right)} \sinh \left(\delta y_{j}\right) \\
& \cdot\left[\bar{u}_{j} \bar{v}_{j} \tanh \left(\delta y_{j}\right)+\delta u_{j} \bar{v}_{j}-\delta v_{j} \bar{u}_{j}-\delta u_{j} \delta v_{j} \operatorname{coth}\left(\delta y_{j}\right)\right],
\end{aligned}
$$

$$
\begin{aligned}
\int_{y_{j}}^{y_{j+1}} & e^{-k_{i j}\left(y_{i}-x\right)} B_{j} C_{j} d x \\
= & \frac{e^{-k_{i j} y_{i}} \cdot e^{k_{i j} \bar{y}_{j}}}{\sinh \left(2 \delta y_{j}\right)} \sinh \left(\delta y_{j}\right) \\
& \cdot\left[\bar{u}_{j} \bar{v}_{j} \tanh \left(\delta y_{j}\right)+\delta v_{j} \bar{u}_{j}-\delta u_{j} \bar{v}_{j}-\delta u_{j} \delta v_{j} \operatorname{coth}\left(\delta y_{j}\right)\right],
\end{aligned}
$$




$$
\begin{aligned}
& \int_{y_{j}}^{y_{j+1}} e^{-k_{i j}\left(y_{i}-x\right)} C_{j} D_{j} d x \\
& =\frac{e^{-k_{i j} y_{i}} \cdot e^{k_{i j} \bar{y}_{j}}}{2 \sinh \left(2 \delta y_{j}\right)} \sinh \left(\delta y_{j}\right)\left[4 \bar{v}_{j}^{2} \tanh \left(\delta y_{j}\right)-\delta H_{2 j}\right] \\
& \int_{y_{j}}^{y_{j+1}} e^{-k_{i j}\left(y_{i}-x\right)} B_{j}^{2} e^{-2 x} d x \\
& =\frac{e^{-k_{i j} y_{i}} \cdot e^{k_{i j} \bar{y}_{j}}}{2\left(k_{i j}-2\right) \sinh \left(2 \delta y_{j}\right)} \sinh \left(\left(k_{i j}-2\right) \delta y_{j}\right) \\
& \int_{y_{j}}^{y_{j+1}} e^{-k_{i j}\left(y_{i}-x\right)} B_{j} D_{j} e^{-2 x} d x \\
& =\frac{e^{-k_{i j} y_{i}} \cdot e^{k_{i j} \bar{y}_{j}}}{\left(k_{i j}-2\right) \sinh \left(2 \delta y_{j}\right)} \sinh \left(\left(k_{i j}-2\right) \delta y_{j}\right) \\
& \quad \cdot\left[\bar{u}_{j} \bar{v}_{j} \tanh \left(\delta y_{j}\right)-\delta u_{j}\right]
\end{aligned}
$$

It thus follows from (127)-(134) that

$$
\begin{aligned}
& P_{1, i}=\sum_{j=0}^{n} \frac{e^{-k_{i j} y_{i}} \cdot e^{k_{i j} \bar{y}_{j}}}{6 \cosh \left(\delta y_{j}\right)}\left[2 k_{i j} \bar{u}_{j} \bar{v}_{j} \sinh ^{2}\left(\delta y_{j}\right) \tanh \left(\delta y_{j}\right)\right. \\
& +2 k_{i j} \delta u_{j} \delta v_{j} \sinh \left(\delta y_{j}\right) \cosh \left(\delta y_{j}\right) \\
& +2 \delta u_{j} \bar{v}_{j} \cosh ^{2}\left(\delta y_{j}\right) \\
& +2 \delta v_{j} \bar{u}_{j} \cosh ^{2}\left(\delta y_{j}\right) \\
& +\delta u_{j} \bar{v}_{j}+\delta v_{j} \bar{u}_{j}-3 \bar{u}_{j} \bar{v}_{j} \tanh \left(\delta y_{j}\right) \\
& \left.+3 \delta u_{j} \delta v_{j} \operatorname{coth}\left(\delta y_{j}\right)\right] \text {, } \\
& P_{2, i}=\sum_{j=0}^{n} \frac{e^{-k_{i j} y_{i}} \cdot e^{k_{i j} \bar{y}_{j}}}{4 \cosh \left(\delta y_{j}\right)}\left[\delta H_{1 j} \cosh ^{2}\left(\delta y_{j}\right)\right. \\
& +4 k_{i j} \bar{u}_{j} \delta u_{j} \sinh ^{2}\left(\delta y_{j}\right) \\
& +2 \bar{u}_{j}^{2} \tanh \left(\delta y_{j}\right)+4 \bar{v}_{j}^{2} \tanh \left(\delta y_{j}\right) \\
& -\delta H_{2 j}-\frac{4}{3} \bar{u}_{j} \bar{v}_{j} \tanh \left(\delta y_{j}\right) \\
& +\frac{8}{3} \delta u_{j} \delta v_{j} \operatorname{coth}\left(\delta y_{j}\right)
\end{aligned}
$$

$$
\begin{aligned}
& \text { We can also write } P_{1, i} \text { and } P_{2, i} \text { as } \\
& \qquad P_{1, i}=\sum_{j=0}^{n} P_{1, i j}, \quad P_{2, i}=\sum_{j=0}^{n} P_{2, i j},
\end{aligned}
$$

where

$$
\begin{aligned}
& {\left[\begin{array}{l}
\frac{1}{6} u_{1} v_{1} e^{y_{1}-y_{i}}, \\
\frac{e^{-k_{i j} y_{i}} \cdot e^{k_{i j} \bar{y}_{j}}}{6 \cosh \left(\delta y_{j}\right)}
\end{array}\right.} \\
& \times\left[2 k_{i j} \bar{u}_{j} \bar{v}_{j} \sinh ^{2}\left(\delta y_{j}\right) \tanh \left(\delta y_{j}\right)\right. \\
& \begin{array}{l}
+2 k_{i j} \delta u_{j} \delta v_{j} \sinh \left(\delta y_{j}\right) \cosh \left(\delta y_{j}\right) \\
+2 \delta u_{j} \bar{v}_{j} \cosh ^{2}\left(\delta y_{j}\right)
\end{array} \\
& +2 \delta v_{j} \bar{u}_{j} \cosh ^{2}\left(\delta y_{j}\right)+\delta u_{j} \bar{v}_{j} \\
& +\delta v_{j} \bar{u}_{j}-3 \bar{u}_{j} \bar{v}_{j} \tanh \left(\delta y_{j}\right) \\
& \left.+3 \delta u_{j} \delta v_{j} \operatorname{coth}\left(\delta y_{j}\right)\right] \text {, } \\
& \text { for } j=1, \ldots, n-1 \text {, } \\
& -\frac{1}{6} u_{n} v_{n} e^{-y_{n}+y_{i}} \quad \text { for } j=n, \\
& \int \frac{1}{4} u_{1}^{2} e^{y_{1}-y_{i}}+\frac{1}{6} u_{1} v_{1} e^{y_{1}-y_{i}}, \quad \text { for } j=0, \\
& \frac{e^{-k_{i j} y_{i}} \cdot e^{k_{i j} \bar{y}_{j}}}{4 \cosh \left(\delta y_{j}\right)} \\
& \times\left[\delta H_{1 j} \cosh ^{2}\left(\delta y_{j}\right)\right. \\
& +4 k_{i j} \bar{u}_{j} \delta u_{j} \sinh ^{2}\left(\delta y_{j}\right)+2 \bar{u}_{j}^{2} \tanh \left(\delta y_{j}\right) \\
& +4 \bar{v}_{j}^{2} \tanh \left(\delta y_{j}\right)-\delta H_{2 j}-\frac{4}{3} \bar{u}_{j} \bar{v}_{j} \tanh \left(\delta y_{j}\right) \\
& +\frac{8}{3} \delta u_{j} \delta v_{j} \operatorname{coth}\left(\delta y_{j}\right) \\
& +\frac{4}{3} \bar{u}_{j} \bar{v}_{j} \cosh \left(\delta y_{j}\right) \sinh \left(\delta y_{j}\right) \\
& +\frac{4}{3} \delta u_{j} \delta v_{j} \cdot \operatorname{coth}\left(\delta y_{j}\right) \cosh ^{2}\left(\delta y_{j}\right) \\
& +\frac{4}{3} k_{i j} \delta u_{j} \bar{v}_{j} \sinh ^{2}\left(\delta y_{j}\right) \\
& \left.+\frac{4}{3} k_{i j} \delta v_{j} \bar{u}_{j} \sinh ^{2}\left(\delta y_{j}\right)\right] \text {, } \\
& \text { for } j=1, \ldots, n-1 \text {, } \\
& \frac{1}{4} u_{n}^{2} e^{-y_{n}+y_{i}}+\frac{1}{6} u_{n} v_{n} e^{-y_{n}+y_{i}} \\
& \text { for } j=n \text {. }
\end{aligned}
$$


The terms $P_{3, i}, P_{4, i}$, and $P_{k, i x}(k=1,2,3,4)$ can be computed in the same way and we have

$$
\begin{array}{ll}
P_{1, i x}=-\sum_{j=0}^{n} k_{i j} P_{1, i j}, & P_{2, i x}=-\sum_{j=0}^{n} k_{i j} P_{2, i j}, \\
P_{3, i x}=-\sum_{j=0}^{n} k_{i j} P_{3, i j}, & P_{4, i x}=-\sum_{j=0}^{n} k_{i j} P_{4, i j} .
\end{array}
$$

The result can be summarized in the following theorem.

Theorem 12. Assume that $\bar{y}_{i}=\xi_{i}, \bar{z}_{i}=\left(\bar{u}_{i}, \bar{v}_{i}\right)=\left(\bar{u}\left(\xi_{i}\right), \bar{v}\left(\xi_{i}\right)\right)$ and $\bar{H}_{i}=\int_{-\infty}^{\xi_{i}}\left(\bar{u}^{2}+\bar{u}_{x}^{2}+\bar{v}^{2}+\bar{v}_{x}^{2}\right) d x$ for $i=1, \ldots, n$ with a multipeakon initial data $\bar{z}=(\bar{u}, \bar{v})$ as given by (87). Then, there exists a global solution $\left(y_{i}, u_{i}, v_{i}, H_{i}\right)$ of (109), (136), and (138) with initial data $\left(\bar{y}_{i}, \bar{u}_{i}, \bar{v}_{i}, \bar{H}_{i}\right)$. On each interval $\left[y_{i}(t), y_{i+1}(t)\right]$, one defines $z(t, x)=(u, v)(t, x)$ as the solution of the Dirichlet problem $z-z_{x x}=0$ with boundary conditions $z\left(t, y_{i}(t)\right)=z_{i}(t)$ and $z\left(t, y_{i+1}(t)\right)=z_{i+1}(t)$ for each time $t$. Thus $z=(u, v)$ is a conservative solution of the modified coupled two-component Camassa-Holm system, which is the multipeakon conservative solution.

\section{Conflict of Interests}

The authors declare that there is no conflict of interests regarding the publication of this paper.

\section{Acknowledgments}

This work was supported in part by the Major State Basic Research Development Program 973 (no. 2012CB215202), the National Natural Science Foundation of China (no. 61134001), and Key Laboratory of Dependable Service Computing in Cyber Physical Society (Chongqing University), Ministry of Education.

\section{References}

[1] Y. Fu, Y. Liu, and C. Qu, "Well-posedness and blow-up solution for a modified two-component periodic Camassa-Holm system with peakons," Mathematische Annalen, vol. 348, no. 2, pp. 415448, 2010.

[2] Y. Fu and C. Qu, "Well posedness and blow-up solution for a new coupled Camassa-Holm equations with peakons," Journal of Mathematical Physics, vol. 50, no. 1, article 012906, 2009.

[3] A. Constantin, "The Hamiltonian structure of the CamassaHolm equation," Expositiones Mathematicae, vol. 15, no. 1, pp. 53-85, 1997.

[4] R. Camassa and D. D. Holm, "An integrable shallow water equation with peaked solitons," Physical Review Letters, vol. 71, no. 11, pp. 1661-1664, 1993.

[5] A. Constantin, "On the scattering problem for the CamassaHolm equation," Proceedings of the Royal Society A: Mathematical, Physical and Engineering Sciences, vol. 457, no. 2008, pp. 953-970, 2001.

[6] H. R. Dullin, G. A. Gottwald, and D. D. Holm, "An integrable shallow water equation with linear and nonlinear dispersion," Physical Review Letters, vol. 87, no. 19, Article ID 194501, 2001.
[7] A. Constantin and D. Lannes, "The hydrodynamical relevance of the Camassa-Holm and Degasperis-Procesi equations," Archive for Rational Mechanics and Analysis, vol. 192, no. 1, pp. 165-186, 2009.

[8] A. Constantin and J. Escher, "Wave breaking for nonlinear nonlocal shallow water equations," Acta Mathematica, vol. 181, no. 2, pp. 229-243, 1998.

[9] A. Constantin, "Existence of permanent and breaking waves for a shallow water equation: a geometric approach," Annales de l'Institut Fourier, vol. 50, no. 2, pp. 321-362, 2000.

[10] A. Bressan and A. Constantin, "Global conservative solutions of the Camassa-Holm equation," Archive for Rational Mechanics and Analysis, vol. 183, no. 2, pp. 215-239, 2007.

[11] H. Holden and X. Raynaud, "Global conservative solutions of the Camassa-Holm equation-a Lagrangian point of view," Communications in Partial Differential Equations, vol. 32, no. 10-12, pp. 1511-1549, 2007.

[12] H. Holden and X. Raynaud, "Periodic conservative solutions of the Camassa-Holm equation," Annales de l'Institut Fourier, vol. 58, no. 3, pp. 945-988, 2008.

[13] H. Holden and X. Raynaud, "Global conservative multipeakon solutions of the Camassa-Holm equation," Journal of Hyperbolic Differential Equations, vol. 4, no. 1, pp. 39-64, 2007.

[14] A. Bressan and A. Constantin, "Global dissipative solutions of the Camassa-Holm equation," Analysis and Applications, vol. 5, no. 1, pp. 1-27, 2007.

[15] L. Tian, Y. Wang, and J. Zhou, "Global conservative and dissipative solutions of a coupled Camassa-Holm equations," Journal of Mathematical Physics, vol. 52, Article ID 063702, 2011.

[16] Y. Wang and Y. Song, "On the global existence of dissipative solutions for the modified coupled Camassa-Holm system," Soft Computing, vol. 17, no. 11, pp. 2007-2019, 2013.

[17] Z. Shen, Y. Wang, H. R. Karimi, and Y. Song, "On the multipeakon dissipative behavior of the modified coupled CamassaHolm model for shallow water system," Mathematical Problems in Engineering, vol. 2013, Article ID 107450, 11 pages, 2013. 


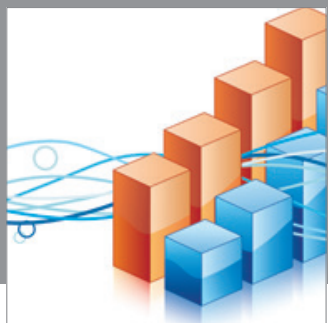

Advances in

Operations Research

mansans

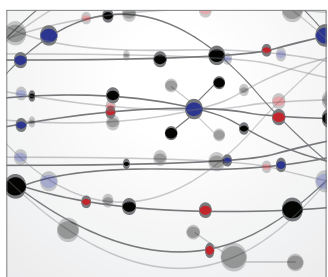

The Scientific World Journal
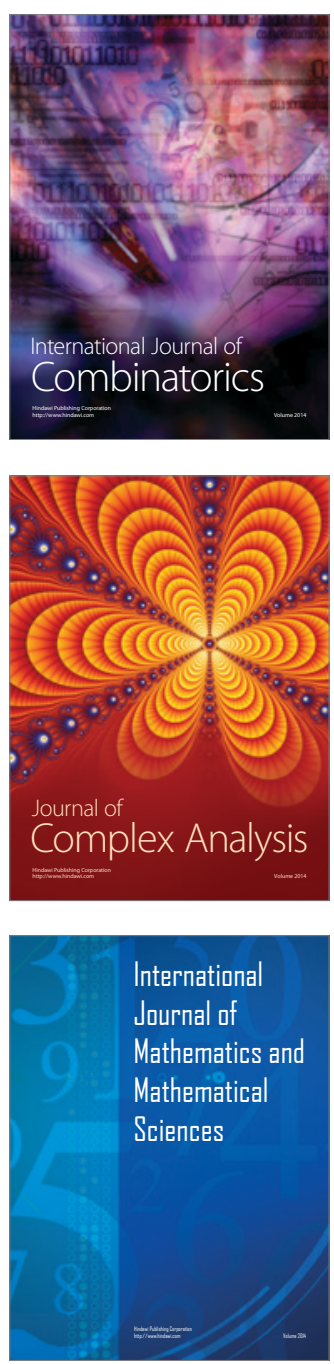
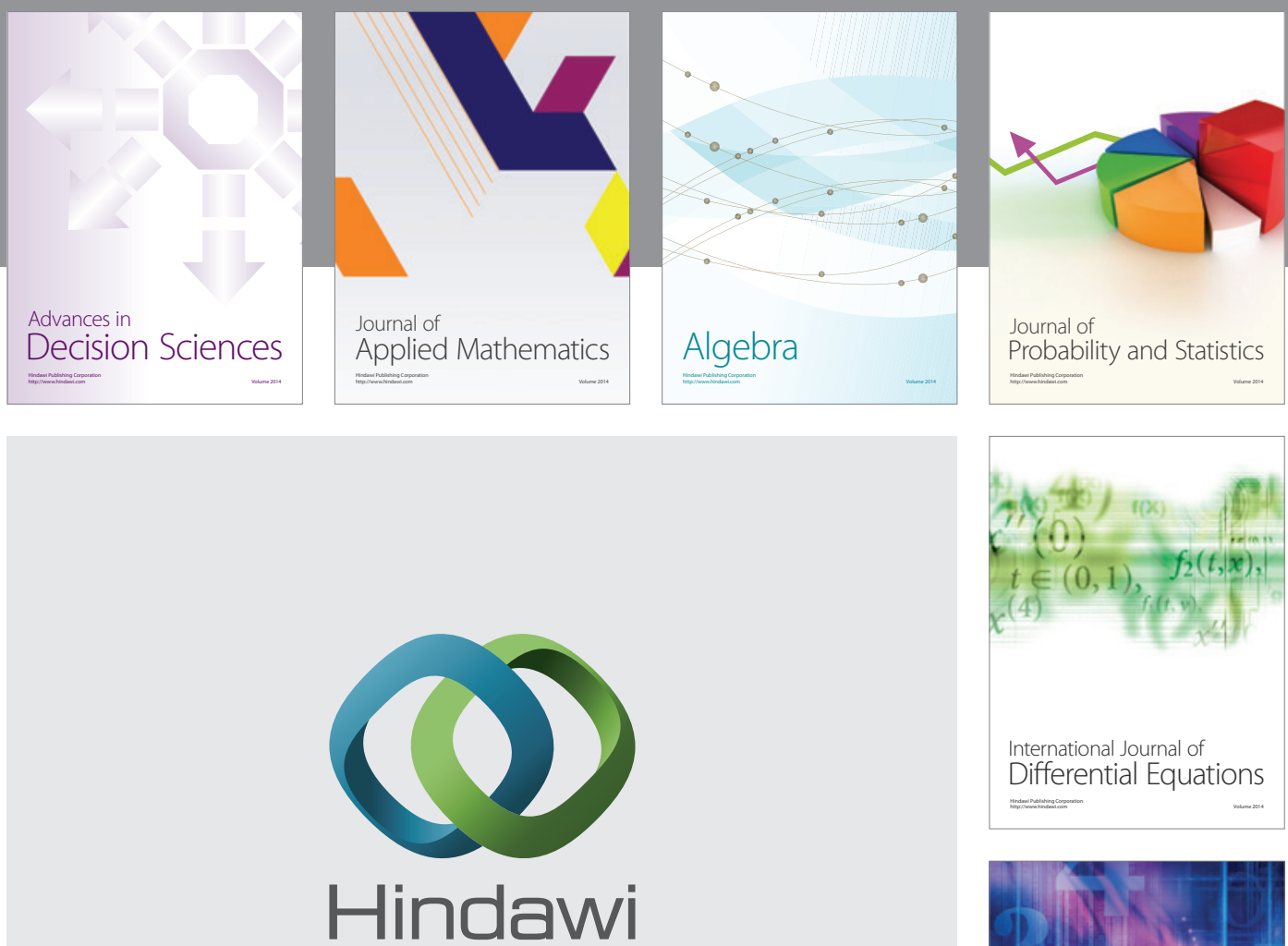

Submit your manuscripts at http://www.hindawi.com
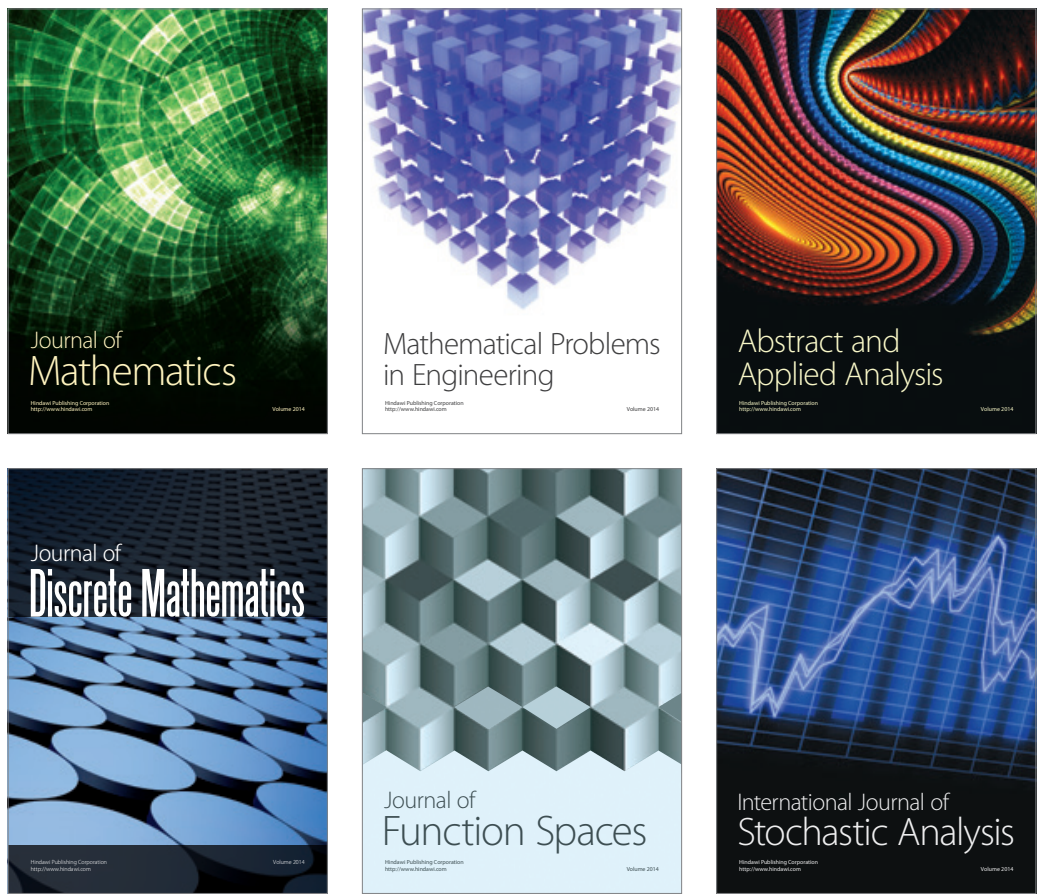

Journal of

Function Spaces

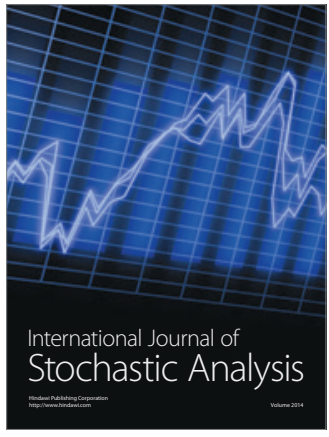

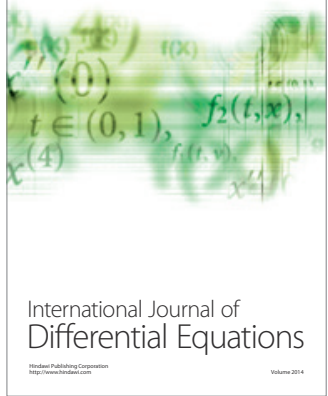
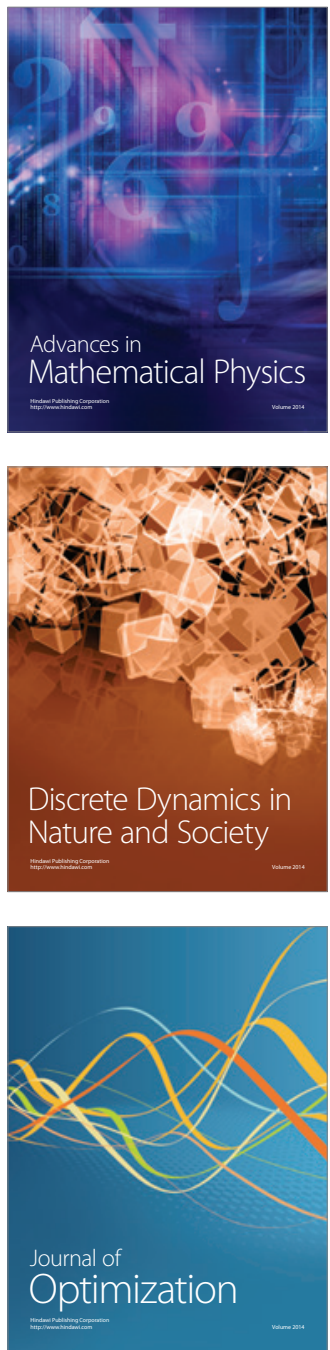